\title{
Rechtsschutz durch den EuGH: Setzt die deutsche Verfassung Schranken?
}

\author{
Prof. Dr. Ekkehart Reimer
}

Institut für Finanz- und Steuerrecht, Universität Heidelberg

Inhaltsübersicht

I. Einleitung

II. Rechtszüge zum EuGH aus verfassungsrechtlicher Sicht

1. Individualrechtsschutz in Steuersachen und das Vorabentscheidungsersuchen (Art. $234 \mathrm{EG)}$

2. EuGH-Verfahren gegen die Bundesrepublik Deutschland

III. Materiell-verfassungsrechtliche Grenzen der negativen Integration

1. Negative Integration des Rechts der direkten Steuern als materielle Vertragsänderung?

2. Wahrung der horizontalen Kompetenzordnung zwi- schen den Gemeinschaftsorganen …........................... 60

3. Wahrung des Gestaltungsraums des mitgliedstaatlichen Gesetzgebers im nichtharmonisierten Bereich 60

IV. Entscheidungsfolgen ........ 71

1. Verwerfung vs. punktuelle Nichtanwendung von Normen 73

2. Zeitliche Wirkung von Entscheidungen 75

3. Umsetzung von EuGHEntscheidungen im System der innerstaatlichen Korrekturvorschriften ....... 79

V. Zusammenfassung 82

\section{Einleitung}

Setzt die deutsche Verfassung dem Rechtsschutz durch den EuGH Schranken? Eine ungewöhnliche Frage! Denn gerade auch aus Sicht des Bundesverfassungsgerichts ist der Europäische Gerichtshof ein gesetzlicher Richter. Dem Rechtsschutz durch den EuGH setzt das 
Grundgesetz deshalb keine Schranken, im Gegenteil: Es erfordert diesen Rechtsschutz. ${ }^{1}$ Aus der Frage spricht also entweder euroskeptisches Wunschdenken oder feine Ironie: Wunschdenken, wenn man mit der Rechtsprechung dieses sich erratisch vortastenden, mit den technischen Untiefen der 27 mitgliedstaatlichen Steuerrechtsordnungen nicht vertrauten Europäischen Gerichtshofs nicht einverstanden ist und nun - auf der Suche nach Rechtsschutz gegen den EuGH - an das Bundesverfassungsgericht denkt. Und feine Ironie, wenn man dieses Wunschdenken aufgreift, um es dann aber gegen die Euroskeptiker zu wenden und den Europäischen Gerichtshof endlich zu der Superrevisionsinstanz mit Normenkontrollbefugnis und umfassendem Zugriff auf das nationale Recht zu erklären, die er - nach traditioneller Lesart ${ }^{2}$ - bislang nicht ist.

Und doch ist das „Kooperationsverhältnis", mit dem das Bundesverfassungsgericht ${ }^{3}$ ebenso wie oberste Gerichtshöfe anderer Mitgliedstaaten 4 den EuGH und sich selbst auf gleiche Augenhöhe $\mathrm{zu}$ bringen scheinen, spannungsreich. ${ }^{5}$ Immer wieder beschert es der Rechtswis-

1 Art. 101 Abs. 1 Satz 2 i.V.m. Art. 19 Abs. 4, Art. 23 Abs. 1 Satz 1 GG und dem Rechtsstaatsprinzip. Hierzu näher unten $36 \mathrm{ff}$.

2 Vgl. nur BFH, Urt. v. 8.11.2000 - I R 6/96, BFHE 193, $399=$ BStB1. II 2001, 570.

3 BVerfG, Urt. v. 12.10.1993 - 2 BvR 2134, 2159/92 - Maastricht, BVerfGE 89, 155 (175); BVerfG, Beschl. v. 7.6.2000 - 2 BvL 1/97 - Bananenmarktordnung, BVerfGE 102, 147 (163, 165).

4 Vgl. etwa die Schlussanträge des Commissaire du gouvernement Guyomar vor dem französischen Conseil d'Etat im Fall Arcelor u.a., RTDE 2007, 378 (389); vgl. auch die Analysen von Pernice, Das Verhältnis europäischer zu nationalen Gerichten im europäischen Verfassungsverbund, Berlin 2006, S. 30 ff., 36 ff.; Mayer/Lenski/Wendel, Der Vorrang des Europarechts in Frankreich, EuR 2008, 63 (80); Oeter, Vielfalt der Gerichte - Einheit des Prozessrechts?, in DGfV Bd. 42 (2007), S. 149 ff.; Grabenzurter, Staatliches Unionsverfassungsrecht, in von Bogdandy (Hrsg.), Europäisches Verfassungsrecht, Heidelberg 2003, S. 283 (284 ff.); jeweils m.w.N. auch zu parallelen Strukturen im Verhältnis zum EGMR.

5 Statt aller Paul Kirchhof, Rechtsschutz durch Bundesverfassungsgericht und Europäischen Gerichtshof, in Merten (Hrsg.), Föderalismus und Europäische Gemeinschaften, Berlin 1990, S. 109 ff.; Paul Kirchhof, Das Kooperationsverhältnis zwischen Bundesverfassungsgericht und Europäischem Gerichtshof, in MüllerGraff (Hrsg.), Perspektiven des Rechts in der Europäischen Union, Heidelberg 1998, S. 163 ff.; Günther Hirsch, Europäischer Gerichtshof und Bundesverfassungsgericht - Kooperation oder Konfrontation?, NJW 1996, 2457; Slaughter/ Stone Sweet/Weiler, The European Courts and National Courts, Oxford 1998; Pernice, Die Dritte Gewalt im Europäischen Verfassungsverbund, EuR 1996, 27; 
senschaft neue Perspektiven und neue Fragen, die gerade für das Steuerrecht von zentraler Bedeutung sind. ${ }^{6}$ Die nachfolgende Bestimmung des Verhältnisses zwischen Bundesverfassungsgericht und Europäischem Gerichtshof geht im Wesentlichen chronologisch vor, wenn sie drei Problemkreise aufgreift:

- Zunächst geht es um die Rechtswege, also den Zugang zu den beiden Gerichten und die prozessrechtlichen Verknüpfungen deutscher Verfahren mit EuGH-Verfahren. ${ }^{7}$

- Ein zweiter Problemkreis betrifft den Inhalt der Entscheidungen des EuGH, mithin die materiellrechtlichen Fragen der Europäischen Integration aus der Sicht des Grundgesetzes, insbesondere die verfassungsrechtlichen Grenzen der negativen Integration in Steuersachen. 8

- Und schließlich richtet sich der Blick auf die Folgen einer Entscheidung des EuGH. Damit sind Fragen auf der Schnittstelle zwischen Prozessrecht und materiellem Recht angesprochen, darunter insbesondere das Problem der zeitlichen Begrenzung von Urteilswirkungen vor und nach der Entscheidung im Fall Meilicke. ${ }^{9}$

Pernice, Das Verhältnis europäischer zu nationalen Gerichten im europäischen Verfassungsverbund, Berlin 2006; Selmer, Die Gewährleistung der unabdingbaren Grundrechtsstandards durch den EuGH, Baden-Baden 1998; Franz C. Mayer, Kompetenzüberschreitung und Letztentscheidung, München 2000; Schwarze, Das "Kooperationsverhältnis" des Bundesverfassungsgerichts mit dem Europäischen Gerichtshof, in Dreier/Badura (Hrsg.), Festschrift 50 Jahre Bundesverfassungsgericht, Tübingen 2001, S. 223 ff.; Scharf, Das Kooperationsverhältnis zwischen Bundesverfassungsgericht und Europäischem Gerichtshof, Diss. Jena 2006; Oeter, Rechtsprechungskonkurrenz zwischen nationalen Verfassungsgerichten, Europäischem Gerichtshof und Europäischem Gerichtshof für Menschenrechte, VVDStRL 66 (2007), 361 (370 ff.); Skouris, Höchste Gerichte an ihren Grenzen - Bemerkungen aus der Perspektive des Gerichtshofes der Europäischen Gemeinschaften, in Hilf/Kämmerer/König (Hrsg.), Höchste Gerichte an ihren Grenzen (2007), S. 19 ff. (37 f.).

6 Hierzu statt aller Offerhaus, Spannungs- oder Kooperationsverhältnis zwischen dem Bundesfinanzhof, dem Bundesverfassungsgericht und dem Gerichtshof der Europäischen Gemeinschaften, in Bottke u.a. (Hrsg.), Recht in Europa. Festgabe zum 30-jährigen Bestehen der Juristischen Fakultät Augsburg, BadenBaden 2003, S. $217 \mathrm{ff}$.

7 Unten II.

8 Unten III.

9 Unten IV. 
Methodisch beschränkt sich die Untersuchung dabei weitgehend auf die spezifischen Interaktionen zwischen innerstaatlichen und Europäischen Institutionen. Gesichtspunkte eines „vertikalen Rechtsvergleichs" zwischen Verfassungsrecht und Verfassungsprozessrecht einerseits und dem Gemeinschaftsrecht andererseits fließen nur punktuell in die Überlegungen ein.

\section{Rechtszüge zum EuGH aus verfassungsrechtlicher Sicht}

Die prozessrechtliche Lage ist geprägt durch den Dualismus von Vorabentscheidungsverfahren (Art. 234 EG $^{10}$ ) und den streitigen Verfahren vor dem EuGH, in denen der Mitgliedstaat als solcher Partei ist ${ }^{11}$ - namentlich den Vertragsverletzungsverfahren (Art. 226 EG12).

Rein quantitativ kommt dabei den Vorabentscheidungsverfahren überragende Bedeutung zu. Von den insgesamt rund 200 EuGH-Verfahren aus dem Bereich der direkten Steuern, die zwischen 1990 und Ende 2008 anhängig gemacht worden sind, stammen $86 \%$ von den nationalen Gerichten (Art. 234 EG), nur gut $13 \%$ gehen auf Vertragsverletzungsverfahren i.S.d. Art. 226 EG zurück.

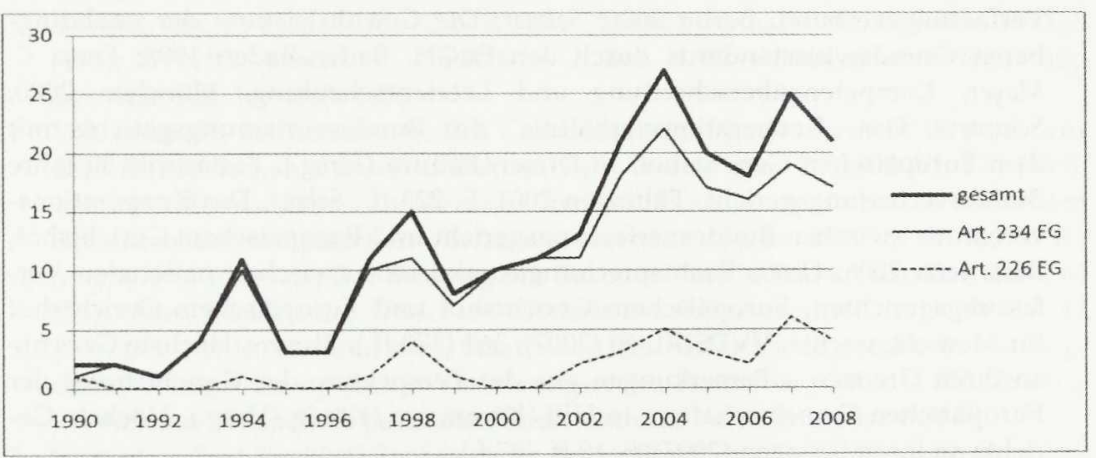

Abb. 1: Zahl der 1990 bis 2008 vor dem EuGH anhängig gemachten Verfahren zu Fragen der direkten Steuern.

10 Dazu unten II 1.

11 Dazu unten II 2.

12 Dazu unten II 2.1. 


\section{Individualrechtsschutz in Steuersachen und das Vorabentscheidungsersuchen (Art. 234 EG)}

Soweit es um Vorabentscheidungsersuchen geht, lautet die Frage: Wie sind die nationalen Rechtsbehelfe und Verfahrensarten in Steuersachen mit den gemeinschaftsrechtlichen Verfahren verknüpft, und welche Bedeutung haben hierfür die Verfassung und das Bundesverfassungsgericht?

Die ganz überwiegende Zahl der EuGH-Verfahren, die eine Steuerfestsetzung durch deutsche Finanzbehörden betreffen, folgen prozessual dem Ablauf, der durch Art. 234 EG vorgegeben ist: Nachdem der außergerichtliche Rechtsschutz aus Sicht eines Beteiligten nicht das gewünschte Ergebnis gebracht hat, wird Klage zum Finanzgericht (oder im Bereich der kommunalen Steuern ausnahmsweise zum Verwaltungsgericht) erhoben.

Prägend für das Verhältnis zwischen Europäischem Gerichtshof und Bundesverfassungsgericht ist - jedenfalls für unseren Bereich ${ }^{13}$ - das Fehlen eines Individualrechtsbehelfs zum EuGH. Über die Scharniernorm des Art. 234 EG$^{14}$ bahnen daher allein die mitgliedstaatlichen Gerichte dem Steuerpflichtigen den Weg zum EuGH.

Allein der BFH hat in den Jahren von 1990 bis 2008 in insgesamt 171 Fällen den EuGH um eine Vorabentscheidung ersucht; und in manchen Jahren machen allein die Vorabentscheidungsersuchen des BFH rund $10 \%$ sämtlicher beim EuGH eingegangenen Vorabentscheidungsersuchen aus 15 - ein Befund, der nebenbei eindrucksvoll die Bedeutung des Steuerrechts als Referenzgebiet für Fragen der Europäischen Integration belegt.

13 Bestandsaufnahme für andere Bereiche und umfassende Reformvorschläge bei Allkemper, Der Rechtsschutz des einzelnen nach dem EG-Vertrag, Baden-Baden 1995; Petzold, Individualrechtsschutz an der Schnittstelle zwischen deutschem und Gemeinschaftsrecht, Baden-Baden 2008.

14 Pernice, Das Verhältnis europäischer zu nationalen Gerichten im europäischen Verfassungsverbund, Berlin 2006, S. 54.

15 Vgl. die Zahlen bei Skouris, Höchste Gerichte an ihren Grenzen - Bemerkungen aus der Perspektive des Gerichtshofes der Europäischen Gemeinschaften, in Hilf/Kämmerer/König (Hrsg.), Höchste Gerichte an ihren Grenzen, Berlin 2007, S. 19 ff. (21 f.). 


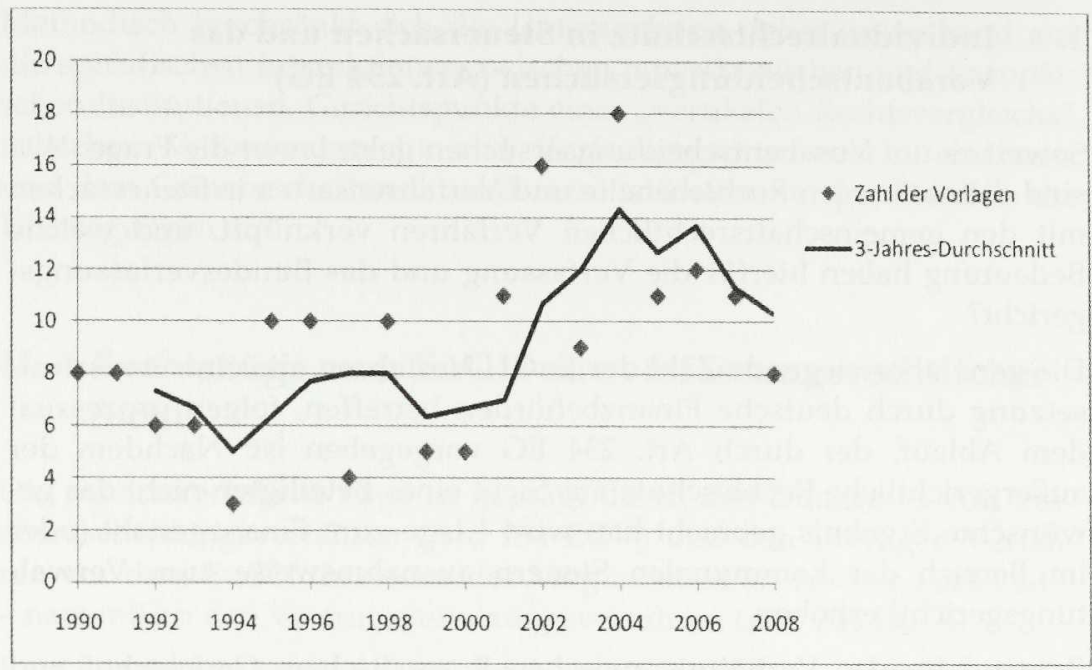

Abb. 2: 1990-2008 beim EuGH eingegangene Vorabentscheidungsersuchen des BFH (inklusive indirekte Steuern und Zölle).

Damit liegt die Zahl der Vorabentscheidungsersuchen des BFH um ein Vielfaches über der Zahl der Fälle, in denen er eine Entscheidung des Bundesverfassungsgerichts im Verfahren der konkreten Normenkontrolle nach Art. 100 Abs. 1 Satz 1 GG eingeholt hat.

\subsection{Ausgangspunkte}

1.1.1 Prinzip des gesetzlichen Richters (Art. 101 Abs. 1 Satz 2 GG)

Auch aus verfassungsrechtlicher Sicht ist dieser Weg bestens trassiert und planiert. Hatte der Zweite Senat des Bundesverfassungsgerichts Anfang der 1970er Jahre noch offen gelassen, ob der EuGH gesetzlicher Richter i.S.d. Art. 101 Abs. 1 Satz 2 GG sei16, deutete sich mit einer Entscheidung aus dem Jahr 1977 eine Änderung der Rechtspre-

16 BVerfG, Beschl. v. 13.10.1970 - 2 BvR 618/68, BVerfGE 29, 198 (207 ff.); BVerfG, Beschl. v. 13.10.1970 - 2 BvR 619/68, BVerfGE 29, 213; BVerfG, Beschl. v. 9.6.1971 - 2 BvR 225/69, BVerfGE 31, 145. 
chung an17, die dann 1986 mit der Entscheidung Solange II vollzogen wurde ${ }^{18}$. Seither ist die Stellung des EuGH als gesetzlicher Richter in der Rechtsprechung des Bundesverfassungsgerichts fest anerkannt. ${ }^{19}$

Das Recht auf den gesetzlichen Richter ist auf Ausfüllung angelegt. Was „gesetzlich“ i.S.d. Art. 101 Abs. 1 Satz 2 GG ist, bestimmt im Hinblick auf den EuGH primär das deutsche Zustimmungsgesetz zu dem alten EWG-Vertrag vom 25.3.1957, der mit Art. 177 EGV die im Wesentlichen gleich lautende Vorläufervorschrift des heutigen Art. 234 EG enthielt. Sekundär sind die durch Art. 101 Abs. 1 Satz 2 GG in Bezug genommenen Vorschriften der Text von Art. 234 EG, das Protokoll über seine Satzung ${ }^{20}$ sowie seine Verfahrensordnung 21 .

Speziell im Hinblick auf den EuGH tritt aber neben Art. 101 Abs. 1 Satz 2 GG eine weitere verfassungsrechtliche Maßstabsnorm hinzu: Mit der Verpflichtung auf Rechtsstaatlichkeit und Gewaltenteilung erfordert auch Art. 23 Abs. 1 GG zwingend einen Zugang der vom Gemeinschaftsrecht Betroffenen zu einem Gemeinschaftsgericht. Nur mit einem EuGH, nicht ohne ihn, darf sich Deutschland nach Art. 23 Abs. 1 GG an dem Projekt der Europäischen Integration beteiligen.

17 BVerfG, Beschl. v. 8.6.1977 - 2 BvR 499/74 und 2 BvR 1042/75; BVerfGE 45, 142: unkommentierte Heranziehung von Art. 101 Abs. 1 Satz 2 GG als Prüfungsmaßstab; Verneinung eines Verstoßes gegen diese Norm allein auf Mangel an Willkür der obersten Bundesgerichte (hier: BVerwG und BGH) gestützt.

18 BVerfG, Beschl. v. 22.10.1986 - 2 BvR 197/83 - Solange II, BVerfGE 73, 339 (366 ff.).

19 BVerfG, Beschl. v. 31.5.1990 - 2 BvL 12, 13/88, 2 BvR 1436/87, BVerfGE 82, 159 (192 f.) - ständige Rechtsprechung; vgl. zuletzt BVerfG, Beschl. v. 4.9.2008 2 BvR 1321/07, DStRE 2009, 60 = HFR 2009, 189 - Rz. 10; BVerfG, Beschl. v. 11.12.2008 - 1 BvR 1563/08, www.bverfg.de - Rz. 10; aus der Literatur statt aller Bethge, in Maunz/Schmidt-Bleibtreu/Klein/Bethge, BVerfGG, Vorb Rz. 358 - Stand Lfg. 28, 2008.

20 Art. 2 ff., 17 ff. des Protokolls über die Satzung des Gerichtshofs der Europäischen Wirtschaftsgemeinschaft vom 17.4.1957, BGB1. II 1957, 1166; heute Protokoll über die Satzung des Gerichtshofs gemäß Artikel 7 des am 26.2.2001 unterzeichneten Vertrags von Nizza zur Änderung des Vertrags über die Europäische Union, der Verträge zur Gründung der Europäischen Gemeinschaften sowie einiger damit zusammenhängender Rechtsakte, ABl. EG Nr. C 80 v. 10.3.2001; geändert durch Beschluss des Rates vom 15.7.2003, ABl. EU Nr. L 188 v. 26.7.2003, 1; und weitere Beschlüsse des Rates.

21 Verfahrensordnung des Gerichtshofs der Europäischen Gemeinschaften v. 19.6.1991, www.curia.europa.eu/de/instit/txtdocfr/txtsenvigueur/txt5.pdf; zuletzt geändert am 23.6.2008, AB1. EU Nr. L 200 v. 29.7.2008, 20. 


\subsubsection{Weitere Anforderungen des Grundgesetzes an den EuGH}

Die Zweispurigkeit verfassungsrechtlicher Maßstabsnormen prägt auch die weiteren Anforderungen, die das Grundgesetz an den Rechtsschutz durch den EuGH stellt: Es ist einerseits das Rechtsstaatsprinzip (einschließlich der Justizgrundrechte und hier namentlich des Begriffs des „Richters" i.S.d. Art. 101 Abs. 1 Satz 2 GG 22 ), andererseits Art. 23 Abs. 1 GG, aus denen sich ein gerichtsverfassungsrechtliches Pflichtenheft für den EuGH und grundlegende Vorgaben für sein Verfahren ergeben. Dieses Pflichtenheft ist zwar weniger detailliert als die einzelnen Justizgrundrechte des Grundgesetzes und die dichte Judikatur des Bundesverfassungsgerichts zu den Anforderungen, die das Rechtsstaatsprinzip innerstaatlich für die Ausgestaltung von Gerichtsorganisation und -verfahren bereithält. Immerhin lassen sich aber eine Reihe einzelner Postulate identifizieren, die die Verfassung auch an den EuGH stellt.

Methodisch kann man dabei - jedenfalls im Sinne einer Mindestgarantie - auf die Rechtsprechung des Bundesverfassungsgerichts zurückgreifen, die bereits Anfang der 1980er Jahre zu den Anforderungen an die Rechtsstaatlichkeit supranationaler und internationaler Spruchkörper ergangen 23 und in Solange II im Hinblick auf den EuGH präzisiert worden ist ${ }^{24}$.

Zu gewährleisten sind damit insbesondere die Neutralität und die persönliche Unabhängigkeit der Richter, mithin zugleich die Existenz von Regelungsmechanismen zur Sicherung der Unbefangenheit zumindest in objektiver Hinsicht, möglicherweise auch bereits zur Abwehr der bloßen Besorgnis der Befangenheit.

$\mathrm{Zu}$ fordern ist weiterhin eine effektive Ausgestaltung des Rechts auf rechtliches Gehör, das mündlich oder schriftlich gewährleistet sein kann. Aus diesem Grundsatz folgt zunächst der schlichte Zugang zu Gericht, der allerdings nicht unmittelbar (im Sinne eines Individualrechtsbehelfs) gewährleistet sein muss 25 ; sodann verlangt er, dass der

22 BVerfG, Beschl. v. 22.10.1986, 2 BvR 197/83 - Solange II, BVerfGE 73, 339 (367).

23 BVerfG, Beschl. v. 10.11.1981 - 2 BvR 1058/79 - Eurocontrol II, BVerfGE 59, 63 (91 f.).

24 BVerfG, Beschl. v. 22.10.1986, 2 BvR 197/83 - Solange II, BVerfGE 73, 339 (367, 371 f., 373 f.).

25 BVerfG, Beschl. v. 10.11.1981 - 2 BvR 1058/79 - Eurocontrol II, BVerfGE 59, 63. 
EuGH seine Gerichtsbarkeit auch ausübt26, dass ein Mindestmaß an prozessualer Gleichheit der Beteiligten gewährleistet ist und dass die Dauer der mündlichen Verhandlung und die Kommunikationsmöglichkeiten vor dem EuGH den sprachlichen Möglichkeiten der Beteiligten und der Komplexität des jeweiligen Verfahrensgegenstandes angemessen sein müssen. Umgekehrt folgt aus Art. 23 Abs. 1 GG aber auch ein Verbot der überlangen Verfahrensdauer. ${ }^{27}$

Dagegen wird sich das Bundesverfassungsgericht wohl nicht dazu verstehen, die Teilnahme und Mitwirkung Deutschlands an der Europäischen Integration davon abhängig zu machen, ob die Geschäftsverteilung innerhalb des EuGH den strengen Maßstäben 28 entspricht, die das Bundesverfassungsgericht dem Art. 101 Abs. 1 Satz 2 GG für die obersten Bundesgerichte entnommen hat 29 .

Im Übrigen erfüllt der EuGH die genannten Vorgaben. Zwar gibt es in der gegenwärtigen Praxis Dysfunktionalitäten; so ist namentlich die Frage gestellt worden, ob die Ausgestaltung der mündlichen Verhandlungen und insbesondere die strikte Zeitdisziplin, die der Gerichtshof den Beteiligten hier abverlangt, ihnen hinreichende Möglichkeiten zur Einwirkung auf das weitere Verfahren und die sachliche Meinungsbildung des Gerichts belässt. Setzt man diese Dysfunktionalitäten allerdings ins Verhältnis zu den spezifischen Sprachschwierigkeiten, die in einer sprachlich nicht homogenen Gemeinschaft unumgänglich sind, verlagern sich die Gewichte nahezu zwangsläufig von der mündlichen Verhandlung auf das schriftliche Verfahren. Die Möglichkeit schriftsätzlicher Äußerungen nach Art. 20 Abs. 2 Verfahrensordnung substituiert dabei Defizite im rechtlichen Gehör in der mündlichen Verhand-

26 BVerfG, Beschl. v. 10.11.1981 - 2 BvR 1058/79 - Eurocontrol II, BVerfGE 59, 63.

27 Vgl. Steger, Überlange Verfahrensdauer bei öffentlich-rechtlichen Streitigkeiten vor deutschen und europäischen Gerichten. Auswirkungen, Ursachen, Abhilfemöglichkeiten, Rechtsschutzmöglichkeiten, Berlin 2008.

28 Hierzu v.a. BVerfGE 17, 294 (299 f.); BVerfGE 95, 322 (330 f.); Morgenthaler in Epping/Hillgruber (Hrsg.), BeckOK GG, Art. 101 GG Rz. 15 ff.

29 Diese Frage wäre zu verneinen: Art. $44 \S \S 2$ und 3 der Verfahrensordnung (Verfahrensordnung des Gerichtshofs der Europäischen Gemeinschaften v. 19.6.1991, www.curia.europa.eu/de/instit/txtdocfr/txtsenvigueur/txt5.pdf; zuletzt geändert am 23.6.2008, ABl. EU Nr. L 200 v. 29.7.2008, 20) stellt die interne Geschäftsverteilung zwischen kleinen und großen Kammern, aber auch innerhalb jeder Gruppe, im Wesentlichen in das freie Ermessen des Plenums. 
lung, so dass bei wertender Betrachtung die Äußerungsmöglichkeiten insgesamt als noch hinreichend erscheinen.

\subsection{Anforderungen an die Annahme eines acte clair}

Aus dem breiten Spektrum der Maßstabsnormen ist und bleibt es aber primär Art. 101 Abs. 1 Satz 2 GG, der das spannungsreiche Verhältnis zwischen den deutschen Gerichten einschließlich des Bundesverfassungsgerichts und dem EuGH konturiert. Hier sollen nun in einem zweiten Anlauf die Inkonsistenzen zwischen der Rechtsprechung des EuGH und des Bundesverfassungsgerichts zu der Frage beschrieben werden, welche tatbestandlichen Konturen die Vorlagepflicht letztinstanzlicher mitgliedstaatlicher Gerichte aus Art. 234 Abs. 3 EG hat.

\subsubsection{Perspektive des Europäischen Gerichtshofs}

Aus Sicht des Gemeinschaftsrechts sind der Verweigerung europäischen Rechtsschutzes durch die nationalen Gerichte enge Grenzen gesetzt. Art. 234 Abs. 3 EG begründet seinem Wortlaut nach eine kategorische, d.h. undurchbrechbare Vorlagepflicht derjenigen mitgliedstaatlichen Gerichte, deren Entscheidungen selbst nicht mehr mit Rechtsmitteln des innerstaatlichen Rechts angefochten werden können. Gleichwohl ist anerkannt, dass in bestimmten eindeutigen Fällen (in actes clairs) auch ein letztinstanzliches mitgliedstaatliches Gericht trotz der Vorgreiflichkeit von Gemeinschaftsrecht für einen bei ihm anhängigen Rechtsstreit von der Vorlage an den EuGH absehen darf. ${ }^{30}$

\subsubsection{Herleitung der Doktrin von acte clair und acte éclairé}

Konstruktiv ist dieser Verzicht auf eine Vorlagepflicht nicht ohne weiteres zu begründen. Denn die Dogmatik des Art. 234 Abs. 3 EG sieht weder Rechtfertigungsmöglichkeiten noch - im Unterschied zu Art. 234 Abs. 2 EG - einen Ermessensspielraum des mitgliedstaatlichen Gerichts vor.

30 Dazu grundlegend Hummert, Neubestimmung der Acte-clair-Doktrin im Kooperationsverhältnis zwischen EG und Mitgliedstaat, Berlin 2006; Herrntann, Die Reichweite der gemeinschaftsrechtlichen Vorlagepflicht in der neueren Rechtsprechung des EuGH, EuZW 2006, 231; Dourado/Palma Borges (Hrsg.), The Acte Clair in EC Direct Tax Law, Amsterdam 2008 - jeweils m.w.N. 
Allerdings könnte sich die Formulierung „eine derartige Frage“ in Art. 234 Abs. 3 EG auf diejenigen Fragen beziehen, für die gem. Art. 234 Abs. 2 EG eine Vorlagekompetenz der nicht-letztinstanzlichen mitgliedstaatlichen Gerichte besteht. Dann umfasste diese Formulierung in Art. 234 Abs. 3 EG das Merkmal „hält [...] eine Entscheidung darüber zum Erlass seines Urteils für erforderlich" aus Art. 234 Abs. 2 EG. In dieser Auslegung des Art. 234 Abs. 3 EG käme also dem jeweiligen letztinstanzlichen Gericht - auf Tatbestandsebene - eine Einschätzungskompetenz im Hinblick auf die Erforderlichkeit der Vorlage $\mathrm{zu}$.

Gegen diese Auslegung spricht aber, dass sie den ersichtlich parallel konstruierten Ausdrücken "eine derartige Frage" in Art. 234 Abs. 2 und Abs. 3 EG eine je unterschiedliche Bedeutung beimisst. Darin allein liegt - selbst wenn man von allen teleologischen und integrationspolitischen Gesichtspunkten absieht - ein meines Erachtens hinreichender Grund gegen die Richtigkeit der hier skizzierten Auslegung.

Insofern überrascht es, dass sich der EuGH in Anlehnung an die Rechtsprechungspraxis des französischen Conseil d'Etat mit der C.I.L.F.I.T.Entscheidung aus dem Jahr 1982 schließlich doch der Auslegung angeschlossen hat, wonach auch im Falle des Art. 234 Abs. 3 EG die Erforderlichkeit der Vorlage von dem jeweiligen mitgliedstaatlichen Gericht geprüft werden dürfe und müsse. ${ }^{31}$

Eindeutig ist die Gedankenführung des EuGH - und damit die Anerkennung der hier skizzierten Argumentation - indes nicht. Denn der Gerichtshof stellt dem Argument von der eigenständigen Prüfungskompetenz der letztinstanzlichen mitgliedstaatlichen Gerichte im Hinblick auf die Erforderlichkeit der Vorlage sogleich den Satz entgegen („dagegen"32), die letztinstanzlichen mitgliedstaatlichen Gerichte seien zur Vorlage verpflichtet, sobald überhaupt - aus ihrer Sicht - „das Gemeinschaftsrecht herangezogen werden muss, um eine Entscheidung des bei ihnen anhängigen Rechtsstreits zu ermöglichen“. Dieser zweite Satz belässt den letztinstanzlichen mitgliedstaatlichen Gerichten eine Prüfungskompetenz, schränkt aber den von ihnen anzulegenden Prü-

31 EuGH, Urt. v. 6.10.1982 - Rs. C-283/81 - Srl CILFIT und Lanificio di Gavardo SpA gg. Ministero della Sanità, EuGHE 1982, 3415 - Rz. 10.

32 EuGH, Urt. v. 6.10.1982 - Rs. C-283/81 - Srl CILFIT und Lanificio di Gavardo SpA gg. Ministero della Sanità, EuGHE 1982, 3415 - Rz. 11. 
fungsmaßstab zunächst ein, indem er nicht mehr auf die Erforderlichkeit einer Klärung durch den EuGH, sondern - viel breiter - auf die bloße Einschlägigkeit von Gemeinschaftsrecht abstellt. Eine gewisse Öffnung ergibt sich dann aber wieder aus der Formulierung, vorzulegen sei „,jede sich stellende Auslegungsfrage“33.

\subsubsection{Unterscheidung von acte clair und acte éclairé}

Im weiteren Verlauf der C.I.L.F.I.T.-Entscheidung formuliert der Gerichtshof - teilweise unter Anknüpfung an seine frühere Rechtsprechung 34 - die beiden zentralen Fallgruppen eines Wegfalls der Vorlagepflicht:

- Erstens entfällt die Vorlagepflicht, wenn „die richtige Anwendung des Gemeinschaftsrechts derart offenkundig [ist], dass keinerlei Raum für einen vernünftigen Zweifel an der Entscheidung der gestellten Frage bleibt" 35 . In dieser ersten Fallgruppe, die als acte clair im engeren Sinne bezeichnet wird, beruht die Offenkundigkeit des Rechtsanwendungsergebnisses - bezogen auf den konkreten Fall - auf einem besonders hohen Maß an Normenklarheit.

- Zweitens entfällt die Vorlagepflicht, „wenn bereits eine gesicherte Rechtsprechung des Gerichtshofs vorliegt, durch die die betreffende Rechtsfrage gelöst ist". Dieser Fall eines acte éclairé liege selbst dann vor, wenn die strittigen Rechtsfragen nicht vollkommen identisch seien. ${ }^{36}$

- Eine Verklammerung beider Fallgruppen stellt der EuGH dadurch her, dass er auch für den Fall des anfänglichen acte clair den mitgliedstaatlichen Gerichten das Gedankenexperiment abverlangt, zu prüfen, ob „auch für die Gerichte der übrigen Mitgliedstaaten und den Gerichtshof die gleiche Gewissheit [über den acte clair] bestünde“37. Nur wenn das letztinstanzliche Gericht hiervon "über-

33 EuGH, Urt. v. 6.10.1982 - Rs. C-283/81 - Srl CILFIT und Lanificio di Gavardo SpA gg. Ministero della Sanità, EuGHE 1982, 3415 - Rz. 11.

34 EuGH, Urt. v. 27.3.1963 - Rs. 28-30/62 - Da Costa, EuGHE 1963, 63 (80, 81).

35 EuGH, Urt. v. 6.10.1982 - Rs. C-283/81 - Srl CILFIT und Lanificio di Gavardo SpA gg. Ministero della Sanità, EuGHE 1982, 3415 - Rz. 16.

36 EuGH, Urt. v. 6.10.1982 - Rs. C-283/81 - Srl CILFIT und Lanificio di Gavardo SpA gg. Ministero della Sanità, EuGHE 1982, 3415 - Rz. 14.

37 EuGH, Urt. v. 6.10.1982 - Rs. C-283/81 - SrI CILFIT und Lanificio di Gavardo SpA gg. Ministero della Sanità, EuGHE 1982, 3415 - Rz. 16. 
zeugt ist", dürfe es auf eine Vorlage nach Art. 234 Abs. 3 EG verzichten.

\subsubsection{Beispiele für das Steuerrecht}

Damit sind für beide Fallgruppen hohe Hürden errichtet, die nur in seltenen Fällen erreicht werden. In der neueren Rechtsprechung des EuGH zu Fragen aus dem Bereich der direkten Steuern finden sich allenfalls einige wenige Fälle, die nach Ergebnis und Begründung exakt prognostizierbar gewesen wären.

Demgegenüber erscheinen mir - anders als Herrn Kollegen Gosch - die mit Marks and Spencer, AMID und Lidl Belgium erkennbaren Leitlinien des EuGH zu den Fragen der grenzüberschreitenden Verlustverrechnung bei weitem noch nicht als geklärt. Gerade da, wo der EuGH für Outbound-Fälle ein nur bedingtes Gebot der "stellvertretenden" Verlustberücksichtigung im Wohnsitzstaat annimmt (Marks and Spencer, Lidl Belgium), sind die Bedingungen für Entstehen und Erlöschen dieses Gebots nicht sicher prognostizierbar. Selbst für ähnlich gelagerte Fälle erfordert Art. 234 Abs. 3 EG daher auch künftig Vorlagen an den EuGH.

Erst recht gilt dies für die vom I. Senat des BFH als acte éclairé angesehene Frage nach der Anwendbarkeit der Kapitalverkehrsfreiheit (Art. 56 Abs. 1 EG) auf wesentliche Beteiligungen an Kapitalgesellschaften, die in Drittstaaten ansässig sind. ${ }^{38}$ Der Senat hat Dividenden, die eine inländische Muttergesellschaft von ihren US-amerikanischen und taiwanesischen Tochtergesellschaften bezogen hatte, dem Schutz der Kapitalverkehrsfreiheit unterstellt. Darin liegt meines Erachtens ein Verstoß gegen Art. 234 Abs. 3 EG. Denn die Rechtsprechung des EuGH ist in diesem Punkt bislang uneinheitlich. Einzelne Entscheidungen zum Europäischen Gesellschaftsrecht sprechen eher für die Anwendbarkeit der Kapitalverkehrsfreiheit, steuerrechtliche Entscheidungen eher dagegen. Vor allem aber verkennt die vom BFH maßgeblich herangezogene "normbezogene Betrachtungsweise" (Anwendbarkeit der Kapitalverkehrsfreiheit, weil die maßgebliche nationale Norm - hier: $§ 8$ b Abs. 5 KStG a.F. - nicht nur wesentliche Beteiligungen,

38 BFH, Urt. v. 26.11.2008 - I R 7/08, DStR 2009, 632 = IStR 2009, 244 m. Anm. Rehm/Nagler. Zur Problematik auch Cordezvener, EG-rechtlicher Grundfreiheitsschutz in der Praxis, IWB Fach 11 EU, Gruppe 2, S. 995 - Heft 10 v. 27.5.2009, S. 497. 
sondern auch bloße Portfoliobeteiligungen erfasst) den kategorialen Unterschied zwischen Anwendungsvorrang und Geltungsvorrang. Richtigerweise hätte der I. Senat des BFH allein auf den konkreten Sachverhalt des Ausgangsverfahrens abstellen dürfen. Der Fall betraf eine beherrschende Beteiligung. Hier herrscht bislang gravierende Rechtsunsicherheit, so dass es eindeutig einer Vorlage an den EuGH bedurft hätte.

\subsubsection{Perspektive des Bundesverfassungsgerichts}

Wenn sich ein oberstes Bundesgericht wie etwa der BFH in Fragen, in denen es auf Gemeinschaftsrecht ankommt und in denen auch die sonstigen Voraussetzungen des Art. 234 Abs. 3 EG erfüllt sind, nicht zu einer Vorlage an den EuGH bereit findet, verletzt es das Recht der Beteiligten auf den gesetzlichen Richter. In diesem Fall könnten der Steuerpflichtige, aber auch das Finanzamt einen Verstoß gegen Art. 101 Abs. 1 Satz 2 GG rügen.

An sich müsste das Bundesverfassungsgericht den Beteiligten in diesem Fall den scheinbar verstellten Weg zum EuGH freiräumen. Genau diesen präzisen Gleichlauf verweigert das Bundesverfassungsgericht aber vielfach. Es entspricht seiner bislang ständigen Rechtsprechung, dass ein Verstoß gegen das Recht auf den gesetzlichen Richter nur dann angenommen wird, wenn die Verweigerung einer Vorlage an den EuGH bei verständiger Würdigung der das Grundgesetz bestimmenden Gedanken als nicht mehr verständlich erscheint und offensichtlich unhaltbar ist. ${ }^{39}$ Diese Selbstbeschränkung des Bundesverfassungsgerichts konkretisiert sich in einer Fallgruppenjurisprudenz, die das Gericht in den letzten Jahren fein ausdifferenziert hat. Die wichtigste Fallgruppe ist dabei diejenige, die das Bundesverfassungsgericht mit dem Schlagwort der Unvollständigkeit der Rechtsprechung bezeichnet. Sie weist drei Untergruppen auf:

- erstens den Fall, dass zu einer entscheidungserheblichen Frage des Gemeinschaftsrechts noch keine einschlägige Rechtsprechung des EuGH vorliegt;

39 BVerfG, Beschl. v. 31.5.1990 - 2 BvL 12, 13/88, 2 BvR 1436/87, BVerfGE 82, 159 (194); BVerfG, Beschl. v. 4.9.2008 - 2 BvR 1321/07, DStRE 2009, $60=$ HFR 2009, 189 - Rz. 10; BVerfG, Beschl. v. 11.12.2008 - 1 BvR 1563/08, www.bverfg.de Rz. 10 und 14; Morgenthaler in Epping/Hillgruber (Hrsg.), BeckOK GG, Art. 101 GG Rz. 23 ff. 
- zweitens den Fall, dass eine zwar vorliegende Rechtsprechung des EuGH die entscheidungserhebliche Frage noch nicht erschöpfend beantwortet hat;

- drittens den Fall, dass eine Fortentwicklung der Rechtsprechung des Europäischen Gerichthofs nicht nur als entfernte Möglichkeit erscheint.

In allen drei Unterfällen sieht das Bundesverfassungsgericht das Recht auf den gesetzlichen Richter (Art. 101 Abs. 1 Satz 2 GG) nur dann als verletzt an, wenn das letztinstanzliche Hauptsachegericht "den ihm in solchen Fällen notwendig zukommenden Beurteilungsrahmen in unvertretbarer Weise überschritten hat" 40 - etwa, weil mögliche Gegenauffassungen zu der entscheidungserheblichen Frage des Gemeinschaftsrechts gegenüber der vom Gericht vertretenen Meinung eindeutig vorzuziehen sind 41 .

Es ist bemerkenswert, dass das Bundesverfassungsgericht in voller Kenntnis und Würdigung der C.I.L.F.I.T.-Rechtsprechung des EuGH ${ }^{42}$ seine eigene Toleranzschwelle weit über das Maß hinaus erhöht, das Art. 234 Abs. 3 EG in der Auslegung des EuGH den letztinstanzlichen mitgliedstaatlichen Gerichten vorgibt. Der Zweite Senat des Bundesverfassungsgerichts hat diese Maßstabsdivergenz im Jahr 1990 mit der Erwägung begründet, das Bundesverfassungsgericht sei kein „Kontrollorgan, das jeden einem (Fach-) Gericht unterlaufenen, die Zuständigkeit des Gerichts berührenden Verfahrensfehler korrigieren müsste". Vielmehr beurteile das Bundesverfassungsgericht die Zuständigkeitsgarantie des Art. 101 Abs. 1 Satz 2 GG als Teil des rechtsstaatlichen Objektivitätsgebots, das auch die Beachtung der Kompetenzregeln fordere, die den oberen Fachgerichten die Kontrolle über die

40 BVerfG, Beschl. v. 31.5.1990 - 2 BvL 12, 13/88, 2 BvR 1436/87, BVerfGE 82, 159 (194 ff.); BVerfG, Beschl. v. 4.9.2008 - 2 BvR 1321/07, DStRE 2009, $60=$ HFR 2009, 189 - Rz. 11.

41 BVerfG, Beschl. v. 31.5.1990 - 2 BvL 12, 13/88, 2 BvR 1436/87, BVerfGE 82, 159 (194 ff.); BVerfG, Beschl. v. 4.9.2008 - 2 BvR 1321/07, DStRE 2009, $60=$ HFR 2009, 189 - Rz. 11.

42 Teils wörtlich zitiert in BVerfG, Beschl. v. 31.5.1990 - 2 BvL 12, 13/88, 2 BvR 1436/87, BVerfGE 82, 159 (193). 
Befolgung der Zuständigkeitsordnung übertrage und auf den Instanzenzug begrenze. ${ }^{43}$

Diese entwaffnend offenen Feststellungen sind ersichtlich aus der Anschauung des Gemeinschaftsrechts als transformiertem, im Range einfachen Bundesrechts geltendem und damit normhierarchisch auf einer Stufe mit GVG, VwGO, ZPO, StPO, FGO, SGG usw. stehendem Völkerrecht geprägt. Sie erinnern damit an die im Elfes-Urteil angelegte Verringerung der verfassungsgerichtlichen Prüfungsdichte im Fall der Urteilsverfassungsbeschwerde auf eine bloße Willkürkontrolle. 44

Im Anwendungsbereich des Gemeinschaftsrechts ist diese Zurückhaltung aber verfehlt. Das folgt aus Art. 23 Abs. 1 Sätze 1 und 2 GG i.V.m. den Zustimmungsgesetzen zu den Römischen Verträgen. Durch diese mitgliedstaatlichen Rechtsakte wird der vom Gemeinschaftsrecht beanspruchte Anwendungsvorrang jedenfalls in der Normallage, d.h. diesseits ausbrechender Rechtsakte, von der Rechtsordnung des Grundgesetzes anerkannt und in sie eingefügt. Daher ist Art. 234 Abs. 3 EG auch innerstaatlich von all denjenigen Gerichten sensu stricto zu beachten, die den Tatbestand dieser Norm erfüllen. Das gilt zunächst für die Beurteilung einer eigenen Vorlagepflicht des Bundesverfassungsgerichts. ${ }^{45}$ Es gilt aber auch für die Anwendung des Art. 101 Abs. 1 Satz 2 GG. Denn gerade wenn man davon ausgeht, dass regelmäßig nicht das Bundesverfassungsgericht, sondern bereits das jeweils höchste Fachgericht dasjenige Gericht ist, „dessen Entscheidungen selbst nicht mehr mit Rechtsmitteln des innerstaatlichen Rechts angefochten werden können" (Art. 234 Abs. 3 EG), muss für den (Ausnahme-) Fall, dass es nach dieser höchsten fachgerichtlichen Entscheidung doch noch zu einer weiteren Entscheidung (hier: des Bundesverfassungsgerichts) kommt, für das Bundesverfassungsgericht derselbe strikte Maßstab gelten wie für das höchste Fachgericht. Die eigene Vorlagepflicht kann man dem Bundesverfassungsgericht in diesem Fall mit Blick auf die prinzipielle Begrenzung seines Prüfungsmaßstabs auf Verfassungsrecht allenfalls dann ersparen, wenn dies nicht auf Kosten des effet utile des Art. 234 Abs. 3 EG geht. Daraus folgt, dass das Bundesverfassungsgericht für den Fall, dass es nicht selbst nach Art. 234

43 BVerfG, Beschl. v. 31.5.1990 - 2 BvL 12, 13/88, 2 BvR 1436/87, BVerfGE 82, 159 (194).

44 BVerfG, Urt. v. 16.1.1957 - 1 BvR 253/56 - Elfes, BVerfGE 6, 32 (43).

45 Dazu im Einzelnen unten II 1.3.4. 
Abs. 3 EG vorlegt, dafür sorgen muss, dass sich jedenfalls das letztinstanzliche Fachgericht im zweiten Durchgang zu einer Vorlage des Falles an den EuGH versteht. Das kann nur durch eine Synchronisierung der Maßstäbe von Art. 234 Abs. 3 EG einerseits und Art. 101 Abs. 1 Satz 2 GG anderseits erreicht werden. Diese Maßstabsgleichheit ist von Gemeinschafts und von Verfassungs wegen geboten.

Im Ergebnis muss das Bundesverfassungsgericht daher seine Prüfungsdichte bei Urteilsverfassungsbeschwerden, die eine Verletzung des Rechts auf den EuGH als den gesetzlichen Richter rügen, auf hundert Prozent erhöhen. Für die Beschränkung auf eine Willkürkontrolle 46 ist hier kein Raum. 47

\subsection{Besonderheiten einzelner Ausgangsverfahren}

\subsubsection{Verwaltungsverfahren}

Die Einzelanalyse der unterschiedlichen Arten von Ausgangsverfahren nimmt zunächst das Verwaltungsverfahren in den Blick. Hier ist zunächst klar: Die Finanzbehörden sind keine Gerichte i.S.d. Art. 234 EG; ihnen fehlt daher die Vorlageberechtigung. Umso drängender stellt sich die Frage, wie sich eine Finanzbehörde verhalten muss, wenn ihr gemeinschaftsrechtliche Bedenken kommen oder wenn derartige Bedenken von einem Steuerpflichtigen plausibel vorgetragen werden.

Verfassungsrechtlicher Ausgangspunkt ist das Legalitätsprinzip (Art. 20 Abs. 3 GG), die Bindung der Verwaltung an Gesetz und Recht. Damit ist zunächst das innerstaatliche Recht, das Steuergesetz, gemeint. Die Finanzbehörde darf und muss das innerstaatliche Recht allerdings aus-

46 Siehe BVerfG, Beschl. v. 31.5.1990 - 2 BvL 12, 13/88, 2 BvR 1436/87, BVerfGE 82, 159 (194); BVerfG, Beschl. v. 4.9.2008 - 2 BvR 1321/07, DStRE 2009, $60=$ HFR 2009, 189 - Rz. 10; BVerfG, Beschl. v. 11.12.2008 - 1 BvR 1563/08, www.bverfg.de - Rz. 10 und 14; Morgenthaler in Epping/Hillgruber (Hrsg.), BeckOK GG, Art. 101 GG Rz. 23 ff.

47 Diesen Maßstab verfehlen noch BVerfG, Beschl. v. 20.9.2007 - 2 BvR 855/06, NJW 2008, 29; BVerfG, Beschl. v. 6.5.2008 - 2 BvR 1830/06, NJW 2008, 2325. Überzeugende Kritik bei Fastenrath, BVerfG verweigert willkürlich die Kooperation mit dem EuGH, NJW 2009, 272. Überzeugend aber die 3. Kammer des 2. Senats des BVerfG, Beschl. v. 25.8.2008 - 2 BvR 2213/06, NVwZ 2009, $515=$ EuGRZ 2008, 633. Noch anhängig ist die Verfassungsbeschwerde des Finanzamts (!) gegen BFH, Urt. v. 26.11.2008 - I R 7/08, DStR 2009, $632=$ IStR 2009, 244. 
legen, und dies nach allen Auslegungsregeln, also unter voller Berücksichtigung des Gebots gemeinschaftsrechtskonformer Auslegung. Speziell im Umsatzsteuerrecht ist das von zentraler Bedeutung.

Jenseits der Wortlautgrenze (wo immer sie liegt) tun sich die Finanzbehörden schwer mit der Beachtung des EG-Rechts, vielleicht weil sie verfassungsrechtlich so gut geschult sind und das Verwerfungsmonopol des Bundesverfassungsgerichts als Schere im Kopf haben. Das Bundesverfassungsgericht hat aber klar ausgesprochen, dass der Anwendungsvorrang des EG-Rechts von allen Behörden und Gerichten $\mathrm{zu}$ beachten, also gerade nicht in Karlsruhe oder Luxemburg monopolisiert ist.

$\mathrm{Zu}$ "Gesetz und Recht" i.S.d. Art. 20 Abs. 3 GG gehören neben den innerstaatlichen Normen auch diejenigen Normen des Gemeinschaftsrechts, die unmittelbar anwendbar sind - also namentlich die Grundfreiheiten, daneben Teile des Richtlinienrechts, soweit die unmittelbare Anwendbarkeit für den Steuerpflichtigen vorteilhaft ist. Zugleich ist die Finanzbehörde durch Art. 20 Abs. 3, Art. 23 Abs. 1 GG an den Satz ${ }^{48}$ gebunden, dass dem Gemeinschaftsrecht Anwendungsvorrang zukommt. Soweit Normen des Gemeinschaftsrechts unmittelbar anwendbar sind und das innerstaatliche Recht frontal gegen sie verstößt, also keine gemeinschaftsrechtskonforme Auslegung mehr möglich ist, muss deshalb bereits das Finanzamt dem Gemeinschaftsrecht zum Durchbruch verhelfen.

Dies kann, muss aber nicht im Billigkeitswege geschehen. Jede Behörde kann gemeinschaftsrechtswidrige Normen des deutschen Rechts auch ohne Rückgriff auf die $\S \S 163,226$ AO unangewendet lassen. An sich bedarf es dazu nicht einmal einer Überzeugung von der Gemeinschaftsrechtswidrigkeit der nationalen Norm; es genügen überwiegende Bedenken.

Allerdings ist hier auch zu berücksichtigen, dass es dann, wenn das Finanzamt zugunsten des Steuerpflichtigen eine Belastungsnorm unangewendet lässt, in der Regel keine spätere gerichtliche Klärung mehr geben kann - der Steuerpflichtige wird nicht klagen, und das Finanz-

48 Des EuGH, Urt. v. 15.7.1964 - Rs. 6/64 - Costa/ENEL, EuGHE 1964, 1151. Grundlegend hierzu Gerhard Hoffmann, Das Verhältnis des Rechts der Europäischen Gemeinschaften zum Recht der Mitgliedstaaten, DÖV 1967, 433 (438); neuerdings Weltr, Inzidente Normverwerfung durch die Exekutive, Berlin 1998, S. 48 ff., 101 ff. 
amt kann nicht klagen. Das Finanzamt hat also keine Möglichkeit mehr, seine Zweifel auszuräumen.

Wenn man hier das Interesse an der Rechtsrichtigkeit und an der Gleichmäßigkeit des Vollzugs in die Überlegungen einstellt, wird man es deshalb in Zweifelsfällen doch für zulässig und geboten ${ }^{49}$ erachten müssen, dass die Finanzbehörde

- die Steuern gemäß dem innerstaatlichen Recht festsetzt,

- ihren Zweifeln aber durch einen Vorläufigkeitsvermerk nach § 165 Abs. 1 Satz 2 Nr. 3 AO (wenn ein BFH- oder EuGH-Verfahren anhängig ist) bzw. § $165 \mathrm{Abs}$. 1 Satz $1 \mathrm{AO}$ (wenn kein derartiges Verfahren anhängig ist) Ausdruck verleiht und

- dem Steuerpflichtigen für den Fall des Einspruchs die Aussetzung der Vollziehung in Aussicht stellt (§361 Abs. 2 Satz 2 AO).

In klaren Fällen einer Gemeinschaftsrechtswidrigkeit kommt sogar eine Aussetzung der Steuerfestsetzung in Betracht (\$ 165 Abs. 1 Satz 4 i.V.m. Satz 1 bzw. Satz 2 Nr. 3 AO).

\subsubsection{Finanzgerichtliches Verfahren}

Weitgehend unproblematisch sind das finanzgerichtliche bzw. das verwaltungsgerichtliche Verfahren. Die Finanzgerichte bzw. die Verwaltungsgerichte müssen nicht nach Art. 234 EG vorlegen, und zwar selbst dann nicht, wenn sie die Revision nicht zulassen, es also nach ihrer eigenen Vorstellung bei dieser einen Instanz bleiben soll. ${ }^{50}$ Denn selbst dann steht dem Steuerpflichtigen noch die Nichtzulassungsbeschwerde ( $\$ 116 \mathrm{FGO}$ ) zu Gebote, über die zwingend der BFH entscheidet ( $\$ 116$ Abs. 5 FGO). Daher ist von vornherein ausgeschlossen, dass das Finanzgericht dadurch, dass es von einer Vorlage an den EuGH nach Art. 234 Abs. 2 EG absieht, das Recht des Steuerpflichtigen auf den gesetzlichen Richter verletzen könnte. ${ }^{51}$

49 Verfehlt ist m.E. die Annahme eines Ermessensspielraums. Zumindest missverständlich Frotscher in Schwarz, AO, § 165 AO Rz. 18 - Stand: 15.4.2008; Cöster in Pahlke/Koenig, AO, München 2004, § 165 AO Rz. 21.

50 Gaitanides in von der Groeben/Schwarze, Kommentar zum EU-/EG-Vertrag, 6. Aufl., Baden-Baden 2003, Art. 234 EG Rz. 64.

51 Zutreffend Morgenthaler in Epping/Hillgruber (Hrsg.), BeckOK GG, Art. 101 GG Rz. 25.1. 
Eindeutig ist umgekehrt, dass die Finanzgerichte alle gemeinschaftsrechtlichen Fragen vorlegen dürfen - und zwar uneingeschränkt, also auch in acte-clair-Fällen. ${ }^{52}$

\subsubsection{Verfahren vor dem Bundesfinanzhof}

1.3.3.1 Bundesfinanzhof als vorlagepflichtiges Gericht i.S.d. Art. 234 Abs. 3 EG

Der Bundesfinanzhof erfüllt den Tatbestand des Art. 234 Abs. 3 EG, wenn man davon ausgeht, dass er selbst - und nicht erst das Bundesverfassungsgericht - in Steuersachen dasjenige deutsche Gericht ist, „dessen Entscheidungen selbst nicht mehr mit Rechtsmitteln des innerstaatlichen Rechts angefochten werden können". Verlangt man für die Auslegung dieses Erfordernisses aus Art. 234 Abs. 3 EG eine konkrete, d.h. auf die Gegebenheiten des jeweiligen Falles bezogene Betrachtungsweise 53 , ist zunächst nur klar, dass das Finanzamt ein Revisionsurteil des BFH nicht mehr mit der Behauptung angreifen kann, es verstoße gegen materielles Verfassungsrecht ${ }^{54}$. Dem Steuerpflichtigen steht dagegen im Unterliegensfall gegen die Entscheidung des BFH noch die Verfassungsbeschwerde zu Gebote; sie ist durchaus ein Rechtsmittel.

Gleichwohl wird in der Literatur - soweit ersichtlich - einhellig die Auffassung vertreten, die Existenz des Rechtsbehelfs Verfassungsbeschwerde führe nicht zu einer Verlagerung der Vorlagepflicht aus Art. 234 Abs. 3 EG von den obersten Fachgerichten auf das Bundesverfassungsgericht, da die Verfassungsbeschwerde lediglich ein außerordentlicher Rechtsbehelf sei. ${ }^{5}$ In dieser Formulierung liegt kein Be-

52 EuGH, Urt. v. 6.10.1982 - Rs. 283/81 - Srl CILFIT und Lanificio di Gavardo SpA gg. Ministero della Sanità, EuGHE 1982, 3415 - Rz. 15.

53 Wegener in Calliess/Ruffert, EUV/EGV-Kommentar, 3. Aufl., München 2007, Art. 234 EG Rz. 24; Schwarze in Schwarze (Hrsg.), EU-Kommentar, Art. 234 EG Rz. 41; Gaitanides in von der Groeben/Schwarze, Kommentar zum EU-/EGVertrag, 6. Aufl., Baden-Baden 2003, Art. 234 EG Rz. 63 m.w.N.

54 Anders im Hinblick auf Verstöße gegen Art. 101 Abs. 1 Satz 2 GG i.V.m. Art. 234 Abs. 3 EG selbst; hierzu siehe unten II 1.3.3.2.

55 Statt aller wiederum Wegener in Calliess/Ruffert, EUV/EGV-Kommentar, 3. Aufl., München 2007, Art. 234 EG Rz. 23; Schwarze in Schwarze (Hrsg.), EUKommentar, Art. 234 EG Rz. 42; Gaitanides in von der Groeben/Schwarze, Kommentar zum EU-/EG-Vertrag, 6. Aufl., Baden-Baden 2003, Art. 234 EG Rz. 64 . 
gründungsersatz; das Ergebnis ist dennoch richtig. Dies ergibt sich allerdings nicht daraus, dass der Verfassungsbeschwerde ein Annahmeverfahren nach $\$ \S 93 \mathrm{a}$ ff. BVerfGG vorgeschaltet ist, das oftmals dazu führt, dass selbst über zulässige Verfassungsbeschwerden nicht in der Sache entschieden wird. Erst recht ist irrelevant, dass Verfassungsbeschwerden außerordentlich selten Erfolg haben. Das Außerordentliche an der Verfassungsbeschwerde ist vielmehr die strikte Begrenzung der Maßstabsnormen auf Verfassungsrecht, genauer: auf spezifisches Verfassungsrecht (Elfes ${ }^{56}$ ). Daher erlaubt das Rechtsmittel der Verfassungsbeschwerde nur eine partielle Überprüfung der angegriffenen Entscheidung des BFH. Insbesondere Fehler bei der Subsumtion des Falles unter die einfachen Steuergesetze können grundsätzlich nicht mehr korrigiert werden. Es ist aber gerade dieses einfache Recht, auf das das Gemeinschaftsrecht zuvörderst einwirkt, das gemeinschaftsrechtskonform auszulegen ist oder dem Anwendungsvorrang des Gemeinschaftsrecht zum Opfer fällt. Würde man die Vorlagepflicht aus Art. 234 Abs. 3 EG erst beim Bundesverfassungsgericht ansiedeln, liefe die Vorlagepflicht deshalb praktisch leer: Denn angesichts des beschränkten Prüfungsmaßstabs des Bundesverfassungsgerichts verlöre die Frage nach der richtigen Auslegung des Gemeinschaftsrechts aus den genannten Gründen ihre Entscheidungserheblichkeit. Damit sprechen nicht nur prozessökonomische Gründe, sondern auch der materielle effet utile des Gemeinschaftsrechts 57 dafür, dass die Pflicht zu Vorabentscheidungsersuchen in steuerrechtlichen Fragen nicht erst das Bundesverfassungsgericht 58 , sondern bereits den BFH trifft 59 .

56 BVerfG, Urt. v. 16.1.1957 - 1 BvR 253/56 - Elfes, BVerfGE 6, 32 (43).

57 Zu seiner Herleitung und Konturierung ausführlich Seyr, Der effet utile in der Rechtsprechung des EuGH, Berlin 2008, S. 100 ff., $272 \mathrm{ff}$.

58 Dazu unten II 1.3.4.

59 Vgl. im Sinne dieser Begründung auch die - allerdings nicht im Kontext von Art. 234 EG stehende - Feststellung des EuGH, Urt. v. 30.9.2003 - Rs. C-224/01 - Gerhard Köbler gegen Republik Österreich, EuGHE 2003, I-10239 - Rz. 34, "dass ein letztinstanzliches Gericht definitionsgemäß die letzte Instanz ist, vor der der Einzelne die ihm aufgrund des Gemeinschaftsrechts zustehenden Rechte geltend machen kann". 


\subsubsection{Folgen einer Verletzung der Vorlagepflicht}

Eine Verletzung der Vorlagepflicht durch den BFH kann drei unterschiedliche Konsequenzen haben:

- Zum einen können der Steuerpflichtige, aus Gründen prozessualer Waffengleichheit aber auch das Finanzamt Verfassungsbeschwerde erheben, gestützt auf Art. 101 Abs. 1 Satz 2 GG. Im Erfolgsfall kommt es zu einer Sachentscheidung des Bundesverfassungsgerichts mit Aufhebung und Zurückverweisung, verbunden mit der Feststellung, dass der BFH zur Vorlage verpflichtet ist. 60 Im zweiten Durchgang würde der Fall dann doch noch den EuGH erreichen.

- Zum andern ist denkbar, dass die Entscheidung des BFH in Rechtskraft erwächst, dann aber nachfolgend in anderer Sache eine einschlägige Entscheidung des EuGH ergeht. In diesem Fall kann der - unterlegene - Steuerpflichtige unter Umständen beanspruchen, dass die zuständige Verwaltungsbehörde den bestands- und sogar rechtskräftigen Steuerbescheid nachträglich noch ändert. Dies ist die prozessuale Situation aus dem Fall Kühne und Heitz ${ }^{61}$, auf den ich im vierten Teil meines Beitrags noch zurückkommen werde ${ }^{62}$.

- Alternativ kommt die Geltendmachung des gemeinschaftsrechtlichen Staatshaftungsanspruchs in Betracht. Dieser Anspruch setzt einen hinreichend qualifizierten Verstoß gegen eine drittschützende Norm des Gemeinschaftsrechts voraus, der für einen Schaden des Steuerpflichtigen unmittelbar kausal geworden ist. ${ }^{63}$ Ein sol-

60 Zur Möglichkeit eines eigenen Vorabentscheidungsersuchens des Bundesverfassungsgerichts an den EuGH siehe unten II 1.3.4.

61 EuGH, Urt. v. 13.1.2004 - Rs. C-453/00 - Kühne und Heitz, EuGHE 2004, I-837.

62 Unten S. $75 \mathrm{ff}$.

63 EuGH, Urt. v. 19.11.1991 - Rs. C-6 und 9/90 - Francovich, EuGHE 1991, I-5357; EuGH, Urt. v. 4.7.2000 - Rs. C-424/97 - Haim, EuGHE 2000, I-5123 - Rz. 36; EuGH, Urt. v. 30.9.2003 - Rs. C-224/01 - Gerhard Köbler gegen Republik Österreich, EuGHE 2003, I-10239 - Rz. 51; st. Rspr. Allgemein zum gemeinschaftsrechtlichen Staatshaftungsanspruch Kischel, Gemeinschaftsrechtliche Staatshaftung zwischen Europarecht und nationaler Rechtsordnung, EuR 2005, 441. 
cher Verstoß kann auch in der Entscheidung eines letztinstanzlichen Gerichts bestehen. 64

\subsubsection{Verfassungsgerichtliches Verfahren}

Im verfassungsgerichtlichen Verfahren gilt - wie gesehen - ein eingeschränkter Prüfungsmaßstab. Prüfungsmaßstab für das Bundesverfassungsgericht ist ausschließlich das Grundgesetz - jedenfalls im Verfassungsbeschwerdeverfahren und bei der Normenkontrolle von Bundessteuergesetzen (Art. 93 Abs. 1 Nr. 2, Art. 100 Abs. 1 Satz 1 Fall 2 GG).

Das heißt aber nicht, dass das Bundesverfassungsgericht nicht selbst an Gemeinschaftsrecht gebunden wäre. Im Gegenteil: Der Anwendungsvorrang des Gemeinschaftsrechts erstreckt sich - soweit es sich um wirksames, d.h. nicht ausbrechendes Gemeinschaftsrecht handelt auch auf das mitgliedstaatliche Verfassungsrecht. 65

Wenn und soweit es nun die Verfassung oder die bisherige Verfassungsinterpretation sind, die gegen Gemeinschaftsrecht verstoßen, ist das Bundesverfassungsgericht auch selbst - außer in Fällen, die sich aus der Sicht des EuGH als acte clair darstellen ${ }^{66}$ - zur Vorlage an den EuGH verpflichtet (Art. 234 Abs. 3 EG i.V.m. Art. 101 Abs. 1 Satz 2 GG). Vorgekommen sind derartige Vorlagen bisher nicht. 67 Denkbar sind sie aber; als Schulbeispiel wird die in Art. 19 Abs. 3 GG angelegte Begrenzung der Grundrechtsberechtigung juristischer Personen auf inländische juristische Personen angeführt ${ }^{68}$. Das Bundesverfassungsgericht erkennt den Art. 234 EG auch für sich selbst als verbindlich an. ${ }^{69}$

64 EuGH, Urt. v. 30.9.2003 - Rs. C-224/01 - Gerhard Köbler gegen Republik Österreich, EuGHE 2003, I-10239 - Rz. 32 ff.

65 Oben II 1.2.2.

66 Hierzu oben II 1.2.1.

67 Hirsch in Schmidt-Aßmann/Sellner/Hirsch/Kemper/Lehmann-Grube (Hrsg.), Festgabe 50 Jahre Bundesverwaltungsgericht, Köln 2003, S. 3, 5.

68 Statt aller Kotzur, Der Begriff der inländischen juristischen Personen nach Art. 19 III GG im Kontext der EU, DÖV 2001, 192; Gosch, Vielerlei Gleichheiten Das Steuerrecht im Spannungsfeld von bilateralen, supranationalen und verfassungsrechtlichen Anforderungen, DStR 2007, 1553 (1556) m.w.N. in Fn. 43.

69 BVerfG, Beschl. v. 29.5.1974 - 2 BvL 52/71 - Solange I, BVerfGE 37, 271 (281 f.); BVerfG, Beschl. v. 25.7.1979 - 2 BvL 6/77, BVerfGE 52, 187 (201) - beide zu Art. 177 EWGV; aus der Literatur statt aller Gaitanides in von der Groeben/Schwarze, Kommentar zum EU-/EG-Vertrag, 6. Aufl., Baden-Baden 2003, Art. 234 EG Rz. 65 m.w.N. 


\subsubsection{Zwischenstaatliche Schiedsverfahren}

Nicht dem Individualrechtsschutz im engeren Sinne zuzuordnen, ihm aber wegen der Befassung mit konkreten Veranlagungsfällen zumindest eng verwandt sind zwischenstaatliche Schiedsverfahren auf der Grundlage der DBA oder des EG-Gewinnberichtigungsübereinkommens. $70 \mathrm{Ob}$ die dort vorgesehenen Schiedskommissionen vorlageberechtigt sind, ist aber insgesamt zurückhaltend zu beurteilen.

Die Vorlageberechtigung scheitert zwar nicht schon daran, dass diese Schiedskommissionen keine rein deutschen Spruchkörper sind. Vielmehr ist durchaus anerkannt, dass auch ein gemeinsames Gericht mehrerer Mitgliedstaaten "Gericht eines Mitgliedstaats" i.S.d. Art. 234 Abs. 2 EG bzw. „einzelstaatliches Gericht“ i.S.d. Art. 234 Abs. 3 EG sein kann. ${ }^{71}$ Die Vorlageberechtigung zwischenstaatlicher Schiedsgerichte kommt dagegen von vornherein nicht in Betracht, wenn auch nur einer der die Schiedskommission mittragenden Staaten (selbst wenn er am konkreten Verfahren nicht beteiligt ist) kein EG-Mitgliedstaat ist und damit nicht der Jurisdiktion des EuGH untersteht. ${ }^{72}$ Schon aus diesem Grund ${ }^{73}$ ist die Schiedsstelle i.S.d. Art. 25 Abs. 5 und 6 DBA USA n.F. nicht vorlageberechtigt.

Im Übrigen ist sorgfältig zu prüfen, ob der zwischenstaatliche Spruchkörper die Anforderungen erfüllt, die der EuGH an den Begriff "Gericht" i.S.d. Art. 234 EG stellt. Zu ihnen zählen insbesondere das Vorliegen einer Rechtsgrundlage, die Dauerhaftigkeit und Unabhängigkeit des Spruchkörpers, der Charakter seiner Gerichtsbarkeit als obligato-

70 Übereinkommen 90/436/EWG v. 23.6.1990/30.7.1990 über die Beseitigung der Doppelbesteuerung im Falle von Gewinnberichtigungen zwischen verbundenen Unternehmen, ABl. EG Nr. L 225/1990, 10 = BGBl. II 1993, 1308; i.d.F. des Übereinkommens v. 21.12.1995, BGB1. II 1999, 1010; und des Protokolls v. 25.5.1999, BGB1. II 1999, 1082.

71 EuGH, Urt. v. 4.11.1997 - Rs. C-337/95 - Dior, EuGHE 1997, I-6013 - Rz. 20 ff., zum Benelux-Gerichtshof.

72 Gaitanides in von der Groeben/Schwarze, Kommentar zum EU-/EG-Vertrag, 6. Aufl., Baden-Baden 2003, Art. 234 EG Rz. 42.

73 Ferner deshalb, weil Nr. 22 des geänderten Protokolls zum DBA USA das Schiedsverfahren grundsätzlich auf einige wenige Maßstabsnormen beschränkt (Art. 4, 5, 7, 9 und 12 DBA USA), die aber für sich genommen gemeinschaftsrechtlich unproblematisch sind; es ist praktisch immer das innerstaatliche Recht, das die Belastungsentscheidung enthält und damit eine mögliche Gemeinschaftsrechtswidrigkeit begründet. 
risch, die Existenz eines kontradiktorischen Verfahrens und die strikte Rechtsbindung.

Den Beratenden Ausschuss i.S.v. Art. 7 des EG-Gewinnberichtigungsübereinkommens ${ }^{74}$ wird man durchaus als Gericht i.S.d. Art. 234 EG ansehen können. Zwar gibt er zunächst lediglich eine Stellungnahme $a b$, die nicht mehr als ein Anhaltspunkt für die Vertragsstaaten ist. Können sich diese aber anschließend nicht innerhalb von sechs Monaten auf eine einvernehmliche Lösung des Rechtsstreits verständigen, wird die Stellungnahme des Beratenden Ausschusses für sie verbindlich. 75

Demgegenüber werden die zwischenstaatlichen Spruchkörper, die gelegentlich in Schiedsklauseln in innergemeinschaftlichen DBA vorgesehen sind, die genannten Voraussetzungen in der Regel schon deshalb nicht erfüllen, weil sie nur einvernehmlich von beiden Vertragsstaaten angerufen werden können. ${ }^{76}$ Zudem beziehen sich die Schiedsklauseln in DBA nur auf Streitigkeiten um die Auslegung und Anwendung der DBA selbst. Da die Verteilungsnormen der DBA sich aber nicht steuerbegründend auswirken, sondern nur - umgekehrt - die nach staatlichem Steuerrecht begründeten Steueransprüche beschränken und damit prinzipiell entlastend wirken, können sie sich in der Regel nicht in Widerspruch zu den Grundfreiheiten setzen. Jedenfalls im Kontext der Grundfreiheiten verliert deshalb die Frage nach der

74 Übereinkommen 90/436/EWG v. 23.6.1990/30.7.1990 über die Beseitigung der Doppelbesteuerung im Falle von Gewinnberichtigungen zwischen verbundenen Unternehmen, ABI. EG Nr. L 225/1990, 10 = BGBI. II 1993, 1308; i.d.F. des Übereinkommens v. 21.12.1995, BGBl. II 1999, 1010; und des Protokolls v. 25.5.1999, BGB1. II 1999, 1082.

75 Art. 12 Abs. 1 Unteabs. 2 EG-Gewinnberichtigungsübereinkommen.

76 So weist z.B. Züger darauf hin, dass es mit Ausnahme des DBA Ägypten/ Niederlande und des DBA Mazedonien/Niederlande keine DBA gebe, die ein obligatorisches, d.h. einseitig durch einen Vertragsstaat initiierbares Schiedsverfahren vorsehen: Züger, Schiedsverfahren für Doppelbesteuerungsabkommen - Möglichkeiten zur Verbesserung des Rechtsschutzes im Internationalen Steuerrecht, Wien 2001, S. 73. Zum Abschluss eines DBA Österreich/Slowakei, dessen Entwurf v. 31.5.2000 (österr. GZ 04 4522/13-IV/4/00; hier zit. nach Züger, Schiedsverfahren für Doppelbesteuerungsabkommen - Möglichkeiten zur Verbesserung des Rechtsschutzes im Internationalen Steuerrecht, Wien 2001, S. 74) eine obligatorische Schiedsklausel enthielt, ist es bislang nicht gekommen. 
Gerichtsqualität von DBA-Schiedskommissionen erheblich an Relevanz.

\subsection{Zwischenergebnis}

Nimmt man alle diese nationalen Verfahren zusammen, drängt sich jedenfalls für den Individualrechtsschutz nur ein Ergebnis auf: Das Grundgesetz setzt dem Rechtsschutz durch den EuGH keine Schranken, sondern erfordert und stimuliert ihn - und zwar auf allen Ebenen.

\section{EuGH-Verfahren gegen die Bundesrepublik Deutschland}

Die Zahl steuerlicher Vertragsverletzungsverfahren gegen Deutschland, die den EuGH erreicht haben, ist bislang gering; in Fragen der direkten Steuern hat die EG-Kommission erst viermal eine entsprechende Klage erhoben ${ }^{77}$. Prozessual stellen sich hier auch kaum Probleme, denen man eine verfassungsrechtliche Dimension zuweisen könnte.

\subsection{Vertragsverletzungsverfahren (Art. 226 EG)}

Namentlich die Vertragsverletzungsverfahren berühren das nationale Prozessrecht und die nationalen Rechtsschutzmöglichkeiten von vornherein nicht; sie betreffen ausnahmslos die Ebene des Gemeinschaftsrechts. Daher sind keine Anhaltspunkte dafür ersichtlich, dass das Grundgesetz einer Anrufung des EuGH durch die Kommission im Verfahren nach Art. 226 EG prozessuale Grenzen setzen könnte. Auch die spätere innerstaatliche Umsetzung der nach Art. 226 EG ergangenen Entscheidungen des EuGH ist verfassungsrechtlich nicht beschränkt oder gehemmt. ${ }^{78}$

77 EuGH, Urt. v. 8.3.2001 - Rs. C-68/99 - Kommission/Deutschland, EuGHE 2001, I-1865, I-1881 - Künstlersozialversicherung; EuGH, Urt. v. 11.9.2007 Rs. C-318/05 - Kommission/Deutschland, EuGHE 2007, I-6957 - Schulgeld; EuGH, Urt. v. 17.1.2008 - Rs. C-152/05 - Kommission/Deutschland, EuGHE 2008, I-39 - Eigenheimzulage; das durch Klageschrift vom 6.6.2007 anhängig gemachte und derzeit noch nicht abgeschlossene Verfahren in der Rechtssache C-269/07 - Riester-Rente.

78 Hierzu näher unten IV. 


\subsection{Zwischenstaatliche Schiedsverfahren (Art. 239 EG)}

Nur wenig anders liegen die Dinge für zwischenstaatliche Schiedsverfahren nach Art. 239 EG, namentlich - ohne dass es bislang Fälle dazu gibt - für Schiedsverfahren auf der Grundlage des Art. 25 Abs. 5 DBA Österreich. Nach dieser Klausel befassen die Vertragstaaten, denen insoweit kein Ermessensspielraum eingeräumt ist, auf Antrag des Steuerpflichtigen den EuGH mit Schwierigkeiten oder Zweifeln bei der Auslegung oder Anwendung dieses Abkommens, wenn ein normales Verständigungsverfahren nicht zum Erfolg geführt hat. Auch hier sind keine Anhaltspunkte dafür ersichtlich, dass das Grundgesetz dem $\mathrm{Zu}$ gang zum EuGH prozessuale Grenzen setzte. Für die spätere innerstaatliche Umsetzung der Schiedssprüche, die der EuGH auf der Grundlage von Art. 239 EG erlassen hat, gilt das oben ${ }^{79}$ Gesagte entsprechend.

\section{Materiell-verfassungsrechtliche Grenzen der negativen Integration}

Zeigen sich in den prozessrechtlichen Fragen also allenfalls geringe Defizite in der verfassungsgerichtlichen Spruchpraxis zu Fragen des Zugangs zum EuGH, aber keinerlei substanzielle Divergenzen in der Rechtsprechung von EuGH einerseits und Bundesverfassungsgericht andererseits, ist die Frage nach den materiellen Grenzen der Integration von einem prinzipiellen, schon in der normativen Fundierung angelegten Antagonismus zwischen verfassungs- und gemeinschaftsrechtlicher Perspektive geprägt.

Die nachfolgende Analyse legt ihren Schwerpunkt auf die verfassungsrechtlichen Grenzen der negativen Integration. ${ }^{80}$ Dabei bezeichnet der Ausdruck "negative Integration" einstweilen alle Entscheidungen des EuGH, die Verbotscharakter gegenüber den Mitgliedstaaten haben, die also den Mitgliedstaaten ein bestimmtes Handeln verbieten. Dem steht der Gegenbegriff der „positiven Integration“ gegenüber, der die ge-

79 Siehe II 2.1.

$80 \mathrm{Zu}$ den gemeinschaftsrechtlichen Begrenzungen siehe aber unten III 2; umfassende theoretische Fundierung bei Ukrow, Richterliche Rechtsfortbildung durch den EuGH, Baden-Baden 1995; neuerdings Konrad Walter, Rechtsfortbildung durch den EuGH, Berlin 2009. 
staltenden und in diesem Sinne politischen Entscheidungen bezeichnet, die der EuGH nicht treffen darf, die vielmehr den Handlungsformen des Art. 249 EG (also vor allem Richtlinie und Verordnung) vorbehalten sind.

Zwar unterliegt auch die positive Integration durch Richtlinien und Verordnungen verfassungsrechtlichen Grenzen. Sie werden aber durch das Prinzip der begrenzten Einzelermächtigung auch in das Gemeinschaftsrecht hineingetragen (Art. 5 Abs. 1 EG); dadurch kommt es insoweit wiederum nur zu graduellen Unterschieden zwischen der verfassungsrechtlichen und der gemeinschaftsrechtlichen Sicht. Zudem sind die Grenzen der positiven Integration für das Steuerrecht - soweit ich sehe - bislang stets beachtet worden. Das alles rechtfertigt es, diesen Fragen hier nicht weiter nachzugehen.

\section{Negative Integration des Rechts der direkten Steuern als materielle Vertragsänderung?}

Für die negative Integration des Rechts der direkten Steuern durch die Rechtsprechung des EuGH lautet die erste Frage: Gibt es Fälle, in denen sie sich als materielle Vertragsänderung darstellt, die man als „ausbrechenden Rechtsakt" im Sinne der Maastricht-Entscheidung des Bundesverfassungsgerichts ansehen kann?

Diese Frage hat der Achte Senat des Bundesfinanzhofs in einem jüngeren AdV-Verfahren aufgeworfen ${ }^{81}$; Gosch hat sie aus der Sicht des erfahrenen Prozessualisten mit überzeugenden Gründen verneint ${ }^{82}$ : Im Bereich des Eingriffsrechts, so seine These, kann der EuGH dem Steuerpflichtigen nur Gutes tun, niemals aber einen Eingriff verschärfen oder ihn gar begründen. Damit fehlt es dem Steuerpflichtigen stets an der Klagebefugnis und an der Verfassungsbeschwerdebefugnis. Umgekehrt ist der Fiskus zwar durch einen ausbrechenden Akt des EuGH zu Gunsten des Steuerpflichtigen beschwert; hier bieten aber weder Grundgesetz noch das Gesetz über das Bundesverfassungsgericht oder die Finanzgerichtsordnung den Fachgerichten die Möglich-

81 BFH, Beschl. v. 14.2.2006 - VIII B 107/04, IStR 2006, 345 (350) m. Anm. Englisch.

82 Gosch, Vielerlei Gleichheiten - Das Steuerrecht im Spannungsfeld von bilateralen, supranationalen und verfassungsrechtlichen Anforderungen, DStR 2007, 1553. 
keit, das Bundesverfassungsgericht mit der Frage zu befassen, ob sich eine Vorabentscheidung des EuGH als ausbrechender Rechtsakt darstellt, denn die Entscheidungen des EuGH sind keine tauglichen Vorlagegegenstände i.S.d. Art. 100 Abs. 1 oder Abs. 2 GG. Jenseits von Art. 101 Abs. 1 Satz 2 GG scheidet auch ein eigener Rechtsschutz des Fiskus zum Bundesverfassungsgericht aus. Insbesondere die Verfassungsbeschwerde kommt nicht in Betracht. Sie scheitert bereits an der Beschwerdeberechtigung, die ihrerseits an die materielle Grundrechtsträgerschaft (die sog. Grundrechtsberechtigung) anknüpft, die der öffentlichen Hand fehlt. Damit ist prozessual in der Tat nicht mehr als ein kleiner Türspalt offen: Die Fachgerichte (Finanzgerichte und BFH) müssten dem EuGH im Anschluss an eine Vorabentscheidung auf eigene Faust die Gefolgschaft versagen, wenn sich die Vorabentscheidung aus ihrer Sicht als ausbrechender Rechtsakt darstellt. Hiergegen kann der unterlegene Steuerpflichtige Verfassungsbeschwerde erheben und auf diese Weise eine Klärung durch das Bundesverfassungsgericht herbeiführen.

Damit ist aber noch nichts über die materiell-verfassungsrechtlichen Grenzen gesagt, denen die Rechtsprechung des EuGH durchaus unterliegt. 83 Materielle Schranken ergeben sich zunächst aus der Souveränität der Bundesrepublik Deutschland, die sich positiv-rechtlich in der durch Art. 20 Abs. 1 und Art. 79 Abs. 3 GG geschützten Staatsqualität niederschlägt, sodann aus dem Demokratieprinzip und dem aus ihm und dem Rechtsstaatsprinzip abgeleiteten Wesentlichkeitsgrundsatz.

83 Hierzu BVerfG, Beschl. v. 22.10.1986 - 2 BvR 197/83 - Solange II, BVerfGE 73, 339; BVerfG, Urt. v. 12.10.1993 - 2 BvR 2134, 2159/92 - Maastricht, BVerfGE 89, 155; BVerfG, Beschl. v. 7.6.2000 - 2 BvL 1/97 - Bananenmarktordnung, BVerfGE 102, 147 (161 ff.); Anm. Christoph Schmid, NJW 2000, 3124. Guter Rechtsprechungsüberblick bei Pernice, Das Verhältnis europäischer zu nationalen Gerichten im europäischen Verfassungsverbund, Berlin 2006, S. 30 ff. Zur Parallelproblematik der ultra-vires-Akte im Unionsrecht BVerfG, Urt. v. 18.7.2005 - 2 BvR 2236/04 - Europäischer Haftbefehl, BVerfGE 113, 273; Anm. Hufeld, JuS 2005, 865. 


\section{Wahrung der horizontalen Kompetenzordnung zwischen den Gemeinschaftsorganen}

Ein für das Steuerrecht bislang nur selten erörterter, aber meines Erachtens zentraler Punkt betrifft das Problem der Gewaltenteilung auf Gemeinschaftsebene. Art. 23 Abs. 1 GG verpflichtet und begrenzt die deutsche Teilnahme am Integrationsprojekt auf Demokratie und Rechtsstaatlichkeit. Auf der Schnittstelle dieser beiden klassischen Verfassungsprinzipien steht das Subprinzip "Gewaltenteilung". Seit Beginn der Europäischen Integration wird dieses Prinzip gemeinschaftsrechtlich zwar ganz anders buchstabiert als im nationalen Verfassungsstaat. Das zeigt sich gerade im Steuerrecht: Initiativberechtigt ist allein die Kommission (Art. 94 EG), als Gemeinschaftsgesetzgeber fungiert bis heute primär der Rat, und die Mitwirkungsmöglichkeiten des Parlaments sind auf Anhörungsrechte begrenzt. Eindeutig zeigt aber Art. 94 i.V.m. 95 Abs. 2 EG, wie sehr das Primärrecht für Gestaltungsentscheidungen im Bereich der direkten Steuern auf die Einstimmigkeit im Rat, d.h. letztlich auf eine vollständige demokratische Legitimation in jedem der 27 Mitgliedstaaten setzt. Aus Sicht von Art. 23 GG ist eine Aufweichung dieses Einstimmigkeitserfordernisses, das gleichermaßen auf horizontale und auf vertikale Gewaltenteilung abzielt, nicht ohne eine Vertragsänderung möglich.

\section{Wahrung des Gestaltungsraums des mitgliedstaatlichen Gesetzgebers im nichtharmonisierten Bereich}

Was folgt nun aus diesem Kombinationsmaßstab von Primärrecht und Verfassungsrecht? Die These lautet: Im nichtharmonisierten Bereich beschränkt sich die Kompetenz des EuGH auf die Identifikation ungerechtfertigter Diskriminierungen und Beschränkungen im mitgliedstaatlichen Steuerrecht, nicht aber auf den Ersatz derartiger mitgliedstaatlicher Regelungen durch neu gestaltete gemeinschaftsrechtskonforme Regelungskonzepte.

Dieser Obersatz markiert die eigentliche Grenze zwischen negativer und positiver Integration; hier liegt der Rubikon. Bei der Auslegung der Grundfreiheiten darf der EuGH nicht positiv integrieren; und umgekehrt geht auch der Spielraum der Mitgliedstaaten für positive Ge- 
staltungsentscheidungen nur bis zu der Grenze, die das wirksam erlassene Primärrecht als das Regelwerk der negativen Integration setzt.

Für das Steuerrecht fällt diese Grenzziehung zwischen negativer und positiver Integration unterschiedlich aus, je nachdem, ob sich die Integration auf Fiskalzwecknormen oder auf steuerliche Lenkungsnormen bezieht.

\subsection{Fiskalzwecknormen}

Fiskalzwecknormen sind Steuernormen, die ausschließlich auf die Erzielung staatlicher Einnahmen ausgerichtet sind. Die individuellen Lasten, die mit der Verfolgung dieses Zwecks notwendig verbunden sind, müssen sie gerecht austeilen. Die Maßstäbe hierfür liefern wiederum das Verfassungs- und das Gemeinschaftsrecht. Der allgemeine und die speziellen Gleichheitssätze des Grundgesetzes verlangen, dass die Lasten nach wirtschaftlicher Leistungsfähigkeit, d.h. insbesondere unter Beachtung zumindest des subjektiven Nettoprinzips unter denjenigen verteilt werden, denen der Gesetzgeber den Status eines Steuerpflichtigen gibt.

Das Gemeinschaftsrecht anerkennt die eigenständige Regelungskompetenz jedes Mitgliedstaats für den Zuschnitt der steuerstaatlichen Solidargemeinschaft, mithin für die Ausgestaltung der persönlichen Steuerpflicht, und für die territoriale Reichweite der Einkommensteuer, mithin für die sachliche Steuerpflicht und die Qualifikation bestimmter Einkunftsquellen als "inländisch" oder ",ausländisch". Wenn und soweit es dadurch kraft mitgliedstaatlicher Setzung zu einer Gleichstellung von Ausländern mit Inländern und/oder von ausländischen Einkünften mit inländischen Einkünften kommt, müssen Steuerinländer und Steuerausländer auch im Hinblick auf ihre Erwerbsaufwendungen (Betriebsausgaben, Werbungskosten) und - als deren Akkumulation und die eventuelle Verlängerung in der Zeit - im Hinblick auf die Verlustverrechnung gleich behandelt werden. Das alles entspricht der ständigen Rechtsprechung des EuGH.

Dieser Rechtsprechung ist an sich auch zu folgen. Uneingeschränkt gilt das für die Feststellung einer tatbestandlichen Diskriminierung. Sie ist die Domäne der negativen Integration am Maßstab des Primärrechts; sie liegt auch in den nichtharmonisierten Teilen des Steuerrechts vollständig in der Kompetenz des EuGH. Die tatbestandliche Diskriminierung schlägt aber nicht automatisch auf den Tenor des Urteils 
durch, beantwortet also noch nicht automatisch die Frage, ob eine bestimmte nationale Regelung gegen die Grundfreiheiten verstößt. Vielmehr kommt der Feststellung der tatbestandlichen Diskriminierung nur indizielle Wirkung zu. Es sind gerade die Beschränkung des EuGH auf die Aufgabe einer negativen Integration und das Verbot einer dem Primärrecht entnommenen positiven Integration, die dazu führen, dass jedenfalls in den nichtharmonisierten Teilen des Steuerrechts nicht jede Diskriminierung - so schwer sie den Einzelnen auch treffen mag gegen die Grundfreiheiten verstößt. Ihren Sitz haben diese Grenzen der primärrechtlichen Integration im Aufbau der Grundfreiheiten auf der Ebene der Rechtfertigungsprüfung erhalten. ${ }^{84}$ Mit dem Rechtfertigungsgrund „Wahrung der ausgewogenen Aufteilung der Besteuerungsbefugnisse", auf den der EuGH in jüngerer Zeit mehrfach zurückgegriffen hat ${ }^{85}$, trägt er den Grenzen der primärrechtlichen Integration meines Erachtens überzeugender Rechnung als in der Vergangenheit.

Was die Vergangenheit angeht, sind die Grenzen der negativen Integration in diesem Bereich möglicherweise nicht immer eingehalten worden; und manches spricht dafür, dass auch die soeben skizzierten neueren Tendenzen in der Rechtsprechung des EuGH noch nicht so ausgereift sind, dass sie bereits eine vollständige Antwort auf die Frage nach der richtigen Abgrenzung der negativen von der positiven

84 Hierzu statt aller Kube, Grenzüberschreitende Verlustverrechnung und die Zuordnung von Verantwortung, IStR 2008, 305.

85 Der Sache nach, zunächst aber noch nicht klar als Rechtfertigungsgrund gefasst, findet sich das Argument bereits in EuGH, Urt. v. 12.5.1998 - Rs. C-336/96 - Gilly, EuGHE 1998, I-2793 - Rz. 24 ff. Als Rechtfertigungsgrund wird es dann klar angesprochen seit EuGH, Urt. v. 13.12.2005 - Rs. C-446/03 Marks \& Spencer, EuGHE 2005, I-10837 - Rz. 43-46. Ebenso nachfolgend: EuGH, Urt. v. 7.9.2006 - Rs. C-470/04 - N gegen Inspecteur van de Belastingdienst Oost, EuGHE 2006, I-7409 - Rz. 41 ff.; EuGH, Urt. v. 12.9.2006 - Rs. C-196/04 - Cadbury Schweppes, EuGHE 2006, I-7995 - Rz. 56; EuGH, Urt. v. 13.3.2007 - Rs. C-524/04 - Test Claimants in the Thin Cap Group Litigation, EuGHE 2007, I-2107 - Rz. 49 ff.; EuGH, Urt. v. 29.3.2007 - Rs C-347/04 - Rewe Zentralfinanz, EuGHE 2007, I-2647 - Rz. 41 ff.; EuGH, Urt. v. 18.7.2007 - Rs. C-231/05 - Oy AA, EuGHE 2007, I-6373 - Rz. 51 ff; EuGH, Urt. v. 8.11.2007 Rs. C-379/05 - Amurta, EuGHE 2007, I-9569 - Rz. 59 ff.; EuGH, Urt. v. 28.2.2008 - Rs. C-293/06 - Deutsche Shell, EuGHE 2008, I-1129 - Rz. 41 ff.; EuGH, Urt. v. 15.5.2008 - Rs. C-414/06 - Lidl Belgium, EuGHE 2008, I-3601 Rz. $31 \mathrm{ff}$. 
Integration sind. Das sei nachfolgend an zwei besonders plastischen Beispielen erläutert.

\subsubsection{Körperschaftsteueranrechnung über die Grenze}

Im Fall Meilicke hat der EuGH die Regelung des alten $\S 36$ Abs. 2 Nr. 3 EStG, wonach nur die Körperschaftsteuer einer unbeschränkt körperschaftsteuerpflichtigen Körperschaft oder Personenvereinigung in Höhe von $3 / 7$ der Einnahmen i.S.d. § 20 Abs. 1 Nr. 1 oder 2 EStG auf die Einkommensteuer angerechnet wird, als mit Art. 56 Abs. 1 EG und Art. 58 Abs. 1 Buchst. a und Abs. 3 EG unvereinbar angesehen und den unbeschränkt steuerpflichtigen Klägern des Ausgangsverfahrens die indirekte Anrechnung ausländischer Körperschaftsteuer zugesprochen. 86

Diese Entscheidung fügt sich bruchlos in die oben skizzierte Diskriminierungsdogmatik (genauer: das Verbot diskriminierender Beschränkungen) ein. Nahezu zwingend erschien nach bisheriger Auffassung auch die Feststellung, dass eine Rechtfertigung nicht gelingen konnte. Genau dies erscheint mir heute aber fraglicher denn je zu sein. Die These lautet: Manninen und Meilicke sind seinerzeit falsch entschieden worden, weil zumindest damals das Sensorium des EuGH für die richtige Grenzziehung zwischen zulässiger negativer und unzulässiger positiver Integration noch nicht vorhanden war und der EuGH daher nicht erkannt hat, dass er mit beiden Urteilen gestaltende, damit positiv-integrierende Entscheidungen getroffen hat.

Die Begründung für die Fehlerhaftigkeit der beiden Urteile setzt bei der Beobachtung an, dass es ja auch den umgekehrten Fall gab - ausländische Aktionäre deutscher Kapitalgesellschaften. Hätten sie ebenfalls die Körperschaftsteueranrechnung oder -erstattung beanspruchen können? Das hätte sich aus der Rechtsprechung des EuGH ebenso gut begründen lassen wie die Entscheidungen in Manninen und Meilicke. Mir scheint aber evident zu sein, dass ein und derselbe Mitgliedstaat nicht in beide Richtungen (Inbound- und Outbound-Dividenden) für Gleichheit sorgen muss. Das wäre schon deshalb unklug, weil es dann - gleiche Konzepte in den jeweils anderen Mitgliedstaaten unterstellt zu Überentlastungen bis hin zur doppelten Körperschaftsteuererstat-

86 EuGH, Urt. v. 6.3.2007 - Rs. C-292/04 - Meilicke, EuGHE 2007, I-1835; vgl. zu einer parallelen Konstellation zuvor bereits EuGH, Urt. v. 7.9.2004 - Rs. C-319/02 - Manninen, EuGHE 2004, I-7477. 
tung käme. Es genügt vielmehr, dass die Diskriminierungsfreiheit in einer Richtung gewährleistet ist; die andere Richtung ist dann Sache des anderen Staates. Im nichtharmonisierten Bereich ist nicht jeder Mitgliedstaat für alles verantwortlich. Es genügt, wenn er ein verallgemeinerbares System verwendet. Im amerikanischen Verfassungsrecht wird hierfür der Begriff der "internal consistency" verwendet. 87

Wenn Deutschland sich entschieden hätte, im Inbound-Fall dem unbeschränkt Steuerpflichtigen die anteilige ausländische Körperschaftsteuer anzurechnen und notfalls auch zu vergüten, darf es sich von Rechts wegen - möglicherweise kontrafaktisch, aber darauf kommt es nicht an - darauf verlassen, dass dies im Outbound-Fall dann der ausländische Ansässigkeitsstaat des Steuerpflichtigen tut. Und umgekehrt: Wenn Finnland bzw. Deutschland im Outbound-Fall für Diskriminierungsfreiheit gesorgt hätten, hätte der EuGH Manninen bzw. Meilicke abweisen müssen.

Hier zeigt sich, dass der EuGH in Manninen und Meilicke eine gestaltende Entscheidung getroffen hat, die eigentlich eine politische Frage gewesen wäre. Politische Fragen sind Harmonisierungsfragen; für sie gilt Art. 94 EG. Der EuGH unterläuft das Einstimmigkeitserfordernis und die horizontale Gewaltenteilung auf Gemeinschaftsebene, wenn er hier am - letztlich zufällig auftauchenden - Einzelfall Festlegungen trifft, die richtlinienersetzenden Charakter haben.

$87 \mathrm{Zu}$ diesem Kriterium als Abgrenzung zwischen (verbotener) Diskriminierung durch eine Rechtsordnung und (nicht verbotener) Disparität zwischen zwei Rechtsordnungen Mason, A Theory of Tax Discrimination (Jean Monnet Working Paper 09/2006, www.jeanmonnetprogram.org/papers/06/060901.pdf); Mason, Flunking the ECJ's Tax Discrimination Test, Columbia Journal of Transnational Law Vol. 46 (2007), 21; Mason, Made in America for European Tax: The Internal Consistency Test, Boston College Law Review Vol. 49 (2008), 1277; Hellerstein, Is ,internal consistency' dead? Reflections on an evolving commerce clause restraint on state taxation, Tax Law Review Vol. 61 (2007), S. 1; ähnlich zuvor bereits Reimer, Die Auswirkungen der Grundfreiheiten auf das Ertragsteuerrecht der Bundesrepublik Deutschland, in Lehner (Hrsg.), Grundfreiheiten im Steuerrecht der EU-Staaten, München 2000, S. 39 ff., 61 f.; Cordewener, Europäische Grundfreiheiten und nationales Steuerrecht, Köln 2002, S. 973 f. 


\subsubsection{Berücksichtigung der Kosten der persönlichen Lebensführung}

Ein ähnlicher Befund ergibt sich bei der Analyse einiger Schlüsselentscheidungen zu Fragen der einkommensteuerlichen Berücksichtigung der Kosten der persönlichen Lebensführung. Im Fall Schumacker hat der EuGH sich darauf festgelegt, dass grundsätzlich der Ansässigkeitsstaat des Steuerpflichtigen zur Berücksichtigung dieser Kosten berufen sei; der Quellenstaat dürfe sie unberücksichtigt lassen. Etwas anderes solle ausnahmsweise gelten, wenn der Steuerpflichtige bei typisierter Betrachtung im Ansässigkeitsstaat ohnehin keine Steuern zahle, weil alle oder nahezu alle seine Einkünfte im Quellenstaat besteuert würden. In diesem Fall, den Deutschland - in Übereinstimmung mit dieser Rechtsprechung des EuGH - mit dem Tatbestand des $§ 1$ Abs. 3 Satz 2 EStG beschreibt - rücke der Quellenstaat gewissermaßen stellvertretend in die Verantwortung des Wohnsitzstaates für die Berücksichtigung der Kosten der persönlichen Lebensführung ein.

Dieses binäre (Alles-oder-Nichts-) Modell lässt sich bei isolierter Betrachtung durchaus sehen. Ein Blick in die Niederlande eröffnet aber interessante Regelungsalternativen, die das Modell des EuGH (dem Deutschland und viele andere Mitgliedstaaten folgen) als beinahe steinzeitlich erscheinen lassen. 88 Der niederländische Normgeber hatte mit einem Besluit voorkoming dubbele belasting von 1989 im Rahmen einer generellen Regelung zur pauschalen Berücksichtigung einer ausländischen Vorbelastung ausländischer Einkunftsteile bei unbeschränkt steuerpflichtigen Arbeitnehmern vorgesehen, dass sich die niederländische Einkommensteuer um den Anteil ermäßigt, den die ausländischen Einnahmen an den Welteinnahmen des Steuerpflichtigen haben. Damit kam es bei Steuerpflichtigen mit ausländischen Einkünften allerdings automatisch auch zu einer anteiligen Reduktion von Steuerverschonungen, namentlich des Grundfreibetrags, familienbezogener Freibeträge und der Abzüge für Unterhaltsverpflichtungen.

Dieses dynamische Modell vermeidet eine Reihe von Dysfunktionalitäten, die unter dem klassischen binären Modell auftreten können. Unter der Annahme, dass auch der oder die jeweils andere(n) Staat(en) parallele Modelle bereitstellen, erweist sich das niederländische Modell als Königsweg zur Sicherung einer exakt einmaligen Berücksich-

88 Hierzu und zum Folgenden EuGH, Urt. v. 12.12.2002 - Rs. C-385/00 - de Groot, EuGHE 2002, I-11819. 
tigung der Kosten der persönlichen Lebensführung unter Wahrung mitgliedstaatlicher Gestaltungsautonomie für den exakten Zuschnitt des Kreises abziehbarer Aufwendungen. Das Modell bietet damit ein in sich geschlossenes System, das den Anforderungen der internal consistency $^{89}$ vollauf genügt.

Allein der EuGH konnte das nach Schumacker nicht mehr so sehen und hat deshalb in seiner Entscheidung des Falles de Groot dem in den Niederlanden ansässigen Steuerpflichtigen, der auch Auslandseinkünfte bezogen hatte, trotzdem den vollen Grundfreibetrag und die vollen weiteren persönlichen und familiären Abzüge zugesprochen. Meines Erachtens ist dies wiederum eine falsche, weil politische Entscheidung. Rechtlich hätte man die Gleichheit im nichtharmonisierten Bereich auch auf der Basis des klugen holländischen Modells erreichen können, weil es dem Maßstab einer inneren Stimmigkeit (internal consistency) des mitgliedstaatlichen Rechts genügt und weil es - hypothetisch zum gemeinschaftsweiten Gesetz gemacht - zu Belastungen führt, die weder Diskriminierungen noch anderweitige Beschränkungen aufwerfen.

\subsubsection{Konsequenzen?}

Dieser Maßstab ist nicht neu 90 , findet aber erst allmählich Verbreitung ${ }^{91}$. Und es wird wohl noch einige Zeit vergehen, bis auch der EuGH seinen neu gewonnenen Respekt für die mitgliedstaatlichen

89 Oben Fußn. 87.

90 Siehe oben Fußn. 87.

91 Außer den in Fußn. 87 Genannten siehe auch Musil, Verfassungs- und europarechtliche Probleme des Alterseinkünftegesetzes, StuW 2005, 278 (286); in Ansätzen auch Lang/Englisch, A European Legal Tax Order Based on Ability to Pay, in Amatucci (Hrsg.), International Tax Law, Den Haag 2006, S. 251 ff., 299 f.; Graetz/Warren, Dividend Taxation in Europe - When the ECJ Makes Tax Policy, CMLR Vol. 44 (2007), 1577 (1620 f.); Bizioli, Balancing the Fundamental Freedoms and Tax Sovereignty, European Taxation 2008, 133 (135). 
Grundentscheidungen im nichtharmonisieren Bereich ${ }^{92}$ so weit präzisiert hat, dass er auch Fälle wie Manninen und Meilicke, Schumacker und de Groot künftig anders entscheidet als in der Vergangenheit.

Die hier interessierende Frage lautet daher: Was sind die Konsequenzen, wenn und solange der EuGH in derartigen Fällen die Rechtfertigung (noch) verneint, also auf einen Verstoß gegen die Grundfreiheiten erkennt? Nach hier vertretener Auffassung wäre ein solches Urteil zwar inhaltlich falsch. Nicht jede Gemeinschaftsrechtswidrigkeit wird man aber sogleich als "ausbrechenden Akt" ansehen können. Auch Richter machen handwerkliche Fehler; das weiß auch das Rechtsstaatsprinzip. Art. 23 Abs. 1 GG gibt dem EuGH einen gewissen Irrtumskredit. Er ist umso größer, je schwieriger die Bedingungen sind, unter denen sich eine in sich schlüssige Rechtsprechung entwickeln muss. Gerade im Bereich "Grundfreiheiten und Steuerrecht" gibt es eine Reihe von Parametern, die die Arbeits- und Entscheidungsbedingungen des Gerichtshofs in der Tat als schwierig erscheinen lassen:

- Die Grundfreiheiten sind normarm; erst allmählich hat sich hier ein Mindestmaß an einheitlicher Dogmatik für Inbound- und Outboundfälle herausgebildet.

- Der Vertragstext enthält keine Rechtfertigungsgründe, die sich passgenau auf Diskriminierungen im Bereich steuerlicher Fiskalzwecknormen anwenden ließen.

92 Der Sache nach, zunächst aber noch nicht klar als Rechtfertigungsgrund gefasst, findet sich das Argument bereits in EuGH, Urt. v. 12.5.1998 - Rs. C-336/96 - Gilly, EuGHE 1998, I-2793 - Rz. 24 ff. Als Rechtfertigungsgrund wird es dann klar angesprochen seit EuGH, Urt. v. 13.12.2005 - Rs. C-446/03 Marks \& Spencer, EuGHE 2005, I-10837 - Rz. 43-46. Ebenso nachfolgend: EuGH, Urt. v. 7.9.2006 - Rs. C-470/04 - N gegen Inspecteur van de Belastingdienst Oost, EuGHE 2006, I-7409 - Rz. 41 ff.; EuGH, Urt. v. 12.9.2006 - Rs. C-196/04 - Cadbury Schweppes, EuGHE 2006, I-7995 - Rz. 56; EuGH, Urt. v. 13.3.2007 - Rs. C-524/04 - Test Claimants in the Thin Cap Group Litigation, EuGHE 2007, I-2107 - Rz. 49 ff.; EuGH, Urt. v. 29.3.2007 - Rs C-347/04 - Rewe Zentralfinanz, EuGHE 2007, I-2647 - Rz. 41 ff.; EuGH, Urt. v. 18.7.2007 - Rs. C-231/05 - Oy AA, EuGHE 2007, I-6373 - Rz. 51 ff; EuGH, Urt. v. 8.11.2007 Rs. C-379/05 - Amurta, EuGHE 2007, I-9569 - Rz. 59 ff.; EuGH, Urt. v. 28.2.2008 - Rs. C-293/06 - Deutsche Shell, EuGHE 2008, I-1129 - Rz. 41 ff; EuGH, Urt. v. 15.5.2008 - Rs. C-414/06 - Lidl Belgium, EuGHE 2008, I-3601 Rz. $31 \mathrm{ff}$. 
- Die Gegenstandsnormen (die Regeln des mitgliedstaatlichen Steuerrechts) weisen oft eine hohe, für mit dem Steuerrecht nicht primär vertraute Juristen (Richter, Generalanwälte) undurchdringliche Komplexität auf. Zudem lassen sie die Eventualitäten von Entscheidungen oft nur mit großer Mühe erkennen.

- Sprachschwierigkeiten und die hohe Beanspruchung des EuGH, namentlich auch der acht Generalanwälte, treten hinzu.

Unter diesen Bedingungen kann sich eine konsistente Rechtsprechung kaum anders als langsam, tastend und in gewisser Weise experimentell entwickeln. Setzt man die Schwelle zu Entscheidungen "ultra vires" hier zu niedrig an, wird die Autorität des Gerichtshofs untergraben. In Gefahr gerät dabei nicht nur die Stabilität der auf Gewaltenteilung angelegten Europäischen Gemeinschaften, sondern auch - auf allen Ebenen - das Vertrauen in die Effektivität und Verlässlichkeit von Rechtsprechung. Dass diese Güter nicht leichtfertig aufs Spiel gesetzt werden dürfen, ist evident.

Richtigerweise wird man die Schwelle, ab der die mitgliedstaatlichen Gerichte und Behörden dem EuGH in der bisherigen Rechtsprechung von Verfassungs wegen die Gefolgschaft versagen dürfen und müssen, hoch anzusetzen haben. Sie ist erst überschritten, wenn der EuGH an seiner Rechtsprechung auch dann festhält, wenn ihm die vorlegenden Gerichte und die zur Stellungnahme berufenen Regierungen der Mitgliedstaaten die Kompetenzgrenzen für den Einzelfall, aber auch in ihrer grundsätzlichen Bedeutung deutlich vorgezeichnet haben, der Gerichtshof sich dennoch notorisch über diese Argumente hinwegsetzt und damit zu erkennen gibt, dass er prinzipiell nicht gewillt ist, den mitgliedstaatlichen Gestaltungsraum im nichtharmonisierten Bereich und die durch das Einstimmigkeitserfordernis aus Art. 94, 95 Abs. 2 EG qualifizierte Organkompetenz des Rates zu respektieren.

\subsection{Lenkungsnormen}

Etwas anders gelagert sind die gemeinschaftsrechtlichen Anforderungen im Bereich der steuerlichen Lenkungsnormen. Während sich im Bereich der Fiskalzwecknormen die Vorgaben der Grundfreiheiten allein auf die Ausgestaltung der Belastungsverteilung beziehen, erstrecken sie sich bei den steuerlichen Lenkungsnormen auch auf die 
mit der Steuerverschärfung bzw. -begünstigung angestrebten Sachzwecke und ihren territorialen Zuschnitt.

Daraus folgt, dass falsche, d.h. von einer richtig verstandenen, den Art. 94 EG berücksichtigenden Gewaltenteilung abweichende Entscheidungen des EuGH im Bereich der steuerlichen Lenkungsnormen doppelte Konsequenzen hätten: Einerseits verändern sie - wie schon im Bereich der reinen Fiskalzwecknormen - die steuerlichen Belastungsentscheidungen und das mitgliedstaatliche Steueraufkommen, anderseits gestalten sie über ihre Lenkungswirkung die außersteuerliche Wirklichkeit. Durch diese Wirkungsverdopplung erscheinen ausbrechende Rechtsakte im Bereich der Lenkungsnormen daher als besonders gravierend.

Zugleich verschiebt sich aber auch die Schwelle zum ausbrechenden Rechtsakt, weil für den Bereich steuerlicher Lenkungsnormen - verglichen mit den reinen Fiskalzwecknormen - eine andere Grenzziehung zwischen negativer und positiver Integration gilt. Einerseits kommt es bei den Lenkungsnormen zu notwendigen Ausweitungen der (Möglichkeiten einer) negativen Integration, weil sich aus selektiven, d.h. auf das Inland bezogenen Steuerbegünstigungen neue Diskriminierungen ergeben, die vor den Grundfreiheiten der Rechtfertigung bedürfen. Andererseits können sich dieselben Lenkungszwecke in der Grundfreiheitsdogmatik als Rechtfertigungsgründe für tatbestandliche Diskriminierungen erweisen oder bestehende Rechtfertigungsgründe verstärken; dadurch verengt sich der Spielraum des EuGH für eine negative Integration auf Basis der Grundfreiheiten.

Exemplarisch lassen sich diese Grenzverschiebungen für das Recht der steuerlichen Gemeinnützigkeit und das Spendenrecht beschreiben. Zutreffend ist in der Literatur immer wieder darauf hingewiesen worden, dass das Gemeinnützigkeitsrecht nicht in der Weise vergemeinschaftet worden ist (und allein auf Basis der Grundfreiheiten auch nicht vergemeinschaftet werden darf), dass ein Mitgliedstaat generell verpflichtet ist, Steuerverschonungen unabhängig von Art und Ort zu gewähren. ${ }^{93}$ Vielmehr steht auch nach den Entscheidungen des EuGH in den

93 Statt aller Peter Fischer, Überlegungen zur Fortentwicklung des steuerlichen Gemeinnützigkeitsrechts, FR 2008, 752; offene Überlegungen bei von Proff, Gemeinnützigkeit nach den "Stauffer"-Urteilen des EuGH und des BFH, IStR 2007, 269 (273); von Proff, Grenzüberschreitende Gemeinnützigkeit nach dem Persche-Urteil des EuGH, IStR 2009, 371. 
Fällen Stauffer ${ }^{94}$ und Persche 95 fest, dass jeder Mitgliedstaat frei ist, die steuerliche Privilegierung auf diejenigen Sachzwecke zu beschränken, die im Inland erfüllt werden und dem Inland zugute kommen. Die Grundfreiheiten gebieten den Mitgliedstaaten lediglich territoriale Konsequenz: Wenn sie die - freie - Grundentscheidung getroffen haben, auch ausländische Sachzwecke zu privilegieren, dürfen sie die Gemeinnützigkeits- und Spendenprivilegien nicht auf die im Inland ansässigen Rechtsträger beschränken.

\subsection{Zwischenergebnis}

Nach diesen Überlegungen ergeben sich unterschiedliche Grenzziehungen, je nachdem, ob die Grundfreiheiten auf reine Fiskalzwecknormen (Lastenausteilungsnormen) oder auf steuerliche Lenkungsnormen einwirken. Speziell für die Fiskalzwecknormen hat eine Sichtung der bisherigen Rechtsprechung eine Reihe von Kompetenzüberschreitungen des EuGH ergeben. Aber selbst in diesen Fällen - also dort, wo der Gerichtshof die Grenzen zulässiger negativer Integration überschritten und in den den Mitgliedstaaten (oder de lege ferenda dem Richtliniengeber) vorbehaltenen Bereich der positiven Integration eingedrungen ist - scheint mir die verfassungsrechtliche Erträglichkeitsschwelle, die Art. 23 Abs. 1 GG vorgibt, noch nicht überschritten zu sein.

Im Gegenteil, es gibt positive Ansätze in der Rechtsprechung des EuGH. Hierzu zählen klassische Figuren wie das sog. Anerkennungsprinzip - also die ständige Tendenz des EuGH, auf Rechtfertigungsebene im Rahmen der Erforderlichkeitsprüfung die Mitgliedstaaten an ihren eigenen Regelungskonzepten festzuhalten ${ }^{96}$-, daneben aber auch

94 EuGH, Urt. v. 14.9.2006 - Rs. C-386/04 - Centro Musicologia di Walter Stauffer, EuGHE 2006, I-8203 = IStR 2006, 675.

95 EuGH, Urt. v. 27.1.2009 - Rs. C-318/07 - Hein Persche, FR 2009, $230=$ DStR 2009, 207. Das Urteil beruht auf dem Vorabentscheidungsersuchen des BFH, Beschl. v. 9.5.2007 - XI R 56/05, BFHE 218, 125 = IStR 2007, 599.

96 Hierzu EuGH, Urt. v. 15.5.1997 - Rs. C-250/95 - Futura/Singer, EuGHE 1997, I-2471 - Rz. 40; EuGH, Urt. v. 17.10.2002 - Rs. C-79/01 - Payroll Data Services, EuGHE 2002, I-8923 - Rz. 37. Aus der Literatur statt aller Dautzenberg, FR 1997, 570 (571); Cordevener, Europäische Grundfreiheiten und nationales Steuerrecht, Köln 2002, S. 636 mit Fn. 954; Droscha/Reinter, Verlagerung der Buchführung in andere EG-Mitgliedstaaten?, DB 2003, 1689 (1694); vgl. auch Reimer, Das Anerkennungsprinzip im Europäischen Ertragsteuerrecht, FR 2007, 217; Waldhoff, Kann es im europäischen Steuerecht ein Anerkennungsprinzip geben?, IStR 2009,386 . 
die erst in jüngerer Zeit spürbar gewachsene Bereitschaft des Gerichtshofs, den Mitgliedstaaten gestaltende Grundentscheidungen (namentlich den territorialen Zuschnitt ihrer Besteuerung und die Aufteilung der Besteuerungshoheit untereinander) zu überlassen und sie lediglich zur folgerichtigen Umsetzung dieser Grundentscheidungen zu verpflichten. ${ }^{97}$

\section{Entscheidungsfolgen}

Ein abschließender Blick richtet sich auf die Entscheidungsfolgen und damit auf eine Debatte, in die seit den Entscheidungen des EuGH in den Fällen Gerritse ${ }^{98}$ und Scorpio 99 , aus anderer Perspektive dann aber vor allem nach der Entscheidung im Fall Meilicke100 Bewegung geraten ist und die bis in die letzten Wochen hinein für Unruhe gesorgt hat ${ }^{101}$.

- Im Fall Gerritse ist es sicher nicht das Bundesverfassungsgericht, vielleicht aber der verfasste Rechtswegestaat, der einige Kapriolen geschlagen und die Entscheidung des EuGH damit um ihren effet utile gebracht hat. 102 Dem Steuerpflichtigen wurde nach der Entscheidung des EuGH die von der ersten Instanz gewährte Veranlagung versagt, weil die Rechtsprechung des EuGH nach Auffassung des BFH neben der (im Fall nicht mehr möglichen) Berücksichti-

97 Hierzu statt aller Kube, EuGH-Rechtsprechung zum direkten Steuerrecht Stand und Perspektiven Schriftenreihe („Vorträge und Berichte" des Zentrums für Europäisches Wirtschaftsrecht an der Universität Bonn, Bd. 171), Bonn 2009, S. 11, $16 \mathrm{ff}$.

98 EuGH, Urt. v. 12.6.2003 - Rs. C-234/01 - Arnoud Gerritse, EuGHE 2003, I-5945.

99 EuGH, Urt. v. 30.10.2006 - Rs. C-290/04 - FKP Scorpio Konzertproduktionen $\mathrm{GmbH}, \mathrm{EuGHE} 2006$, I-9461.

100 EuGH, Urt. v. 6.3.2007 - Rs. C-292/04 - Meilicke, EuGHE 2007, I-1835.

101 Exemplarisch genannt sei der Disput zwischen Osterloh und Skouris um den Vortrag von Osterloh, Die Beschränkung der Urteilswirkungen in der Rechtsprechung des EuGH und des Bundesverfassungsgerichts (Bundesfinanzministerium Berlin, 18.11.2008; Veröffentlichung in Vorbereitung).

102 BFH, Urt. v. 10.1.2007 - I R 87/03, IStR 2007, 405; vgl. auch Grants, Anm. zum Schlussurteil in Sachen "Gerritse", IStR 2007, 408; differenzierend Huber, Judicial cooperation and taxpayer protection - effective implementation of ECJ preliminary rulings at the example of the case C-234/01 "Gerritse", in: Legal Remedies in Europen Tax Law (im Druck). 
gung der Betriebsausgaben beim Steuerabzug nur eine nachträgliche Erstattung erfordert. Der Fall Gerritse ist deshalb nun vor dem Bundesverfassungsgericht anhängig. 103

- In Scorpio hat der BFH trotz der Entscheidung des EuGH den gegen den Steuerpflichtigen gerichteten Haftungsbescheid nicht aufgehoben. Es sei nicht erkennbar, dass Künstler der Klägerin zum Zeitpunkt der Entstehung des Steuerabzugs eine Mitteilung über seine Aufwendungen gemacht hätte; die nachträgliche Mitteilung von Betriebsausgaben könne nicht mehr berücksichtigt werden. Außerdem könne der ausländische Vergütungsgläubiger die Aufwendungen selbst im Erstattungsverfahren geltend machen 104 .

- Anlässlich des Falles Meilicke ist - neben den materiellrechtlichen Fragen 105 - die Frage nach der Durchbrechung der Bestandskraft und dem Vertrauensschutz gegen den Richter gestellt worden. Die Bundesregierung hatte vor dem EuGH den Antrag auf eine Begrenzung der zeitlichen Wirkungen der Entscheidung gestellt, um nicht über den Anlassfall hinaus ausländischen Aktionären die deutsche Körperschaftsteuer für die Vergangenheit anrechnen oder erstatten zu müssen. Diesem Antrag hat die Große Kammer des Gerichtshofs - gegen den Schlussantrag von Generalanwalt Tizzano vor der ursprünglich zuständigen Ersten Kammer des Gerichts-

103 Aktenzeichen des BVerfG: 2 BvR 1178/07.

104 BMF, Schr. v. 5.4.2007 - IV C 8 - S 2411/07/0002, BStBI. I 2007, 449; zu den Auswirkungen der nachfolgenden Entscheidung des EuGH (EuGH, Urt. v. 15.2.2007 - Rs. C-345/04 - Centro Equeste da Leziria Grande, EuGHE 2007, I-1425) auch OFD Koblenz, Vfg. v. 19.12.2007 - S 2302 A - St 33 3, juris. Aus der Literatur zu den damit verbundenen Fragen Schön, Quellenbesteuerung in Europa, JbFfSt 2007/2008, S. 89 ff.; Cordenener/Grams/Molenaar, Neues aus Luxemburg zur Abzugsbesteuerung nach § 50a EStG - Erste Erkenntnisse aus dem EuGH-Urteil vom 3.10.2006 (Scorpio), IStR 2006, 739; Intemann/Nacke, Die EuGH-Entscheidung in der Rs. Scorpio - Anmerkung zum BMF-Schreiben vom 5.4.2007, DB 2007, 1430; Eicker, Anm. zu EuGH, Urt. v. 30.10.2006 (Scorpio), BB 2007, 358; Kofler, Scorpio: "Ausländersteuer" und Gemeinschaftsrecht, ÖStZ 2007, 79; Pistone, Three Decisions that Did ... Not Change the Future of European Taxes, Intertax 2006, 582; Kempermann, Besteuerung beschränkt steuerpflichtiger Künstler und Sportler - Zur geplanten Neuregelung der $\S \S 50,50$ a EStG, FR 2008, 591.

105 Hierzu oben III 3.1.1. 
hofs 106 , aber in Übereinstimmung mit dem nachfolgenden Schlussantrag von Generalanwältin Stix-Hackl107 - nicht stattgegeben 108 .

Im Zentrum der nachfolgenden Analyse stehen die Fragen, welche sachliche Streubreite eine Entscheidung des EuGH jeweils hat ${ }^{109}$, welche zeitlichen Wirkungen ihr zukommen 110 und inwieweit sie mit dem Instrumentarium der Korrekturvorschriften der Abgabenordnung verfahrensrechtlich umgesetzt werden können 111 .

\section{Verwerfung vs. punktuelle Nichtanwendung von Normen}

Das Bundesverfassungsgericht kann verfassungswidrige Normen verwerfen, d.h. sie erga omnes und mit Rückwirkung für nichtig erklären. Diese Normverwerfungskompetenz ist gedanklich die zwingende Folge aus dem Vorrang der Verfassung (Art. 20 Abs. 3 GG), der ein Geltungsvorrang ist: Regelungen, die mit höherrangigem Recht nicht in Einklang stehen, sind grundsätzlich von Anfang an unwirksam, wenn nicht das höherrangige Recht selbst ausnahmsweise (wie in $\S 43$ VwVfG, § 124 Abs. 2 AO) so etwas wie Bestandskraft, d.h. Wirksamkeit trotz Rechtswidrigkeit, anordnet; aber darauf kommt es hier nicht an.

Ganz anders ist die Wirkung der Entscheidungen des EuGH: Sie können nicht weiter gehen als das Gemeinschaftsrecht selbst. Dem Gemeinschaftsrecht kommt nach seinem Selbstanspruch kein Geltungsvorrang zu. Vielmehr beansprucht es lediglich Anwendungsvorrang. Verstöße nationaler Regelungen aller Stufen gegen Gemeinschaftsrecht führen deshalb lediglich dazu, dass die nationale Regelung in dem konkreten Fall unanwendbar ist. 112

106 Schlussanträge des Generalanwalts Tizzano, EuGH v. 10.11.2005 - Rs. C-292/04 - Meilicke, EuGHE 2007, I-1835.

107 Schlussanträge der Generalanwältin Stix-Hackl, EuGH v. 5.10.2006 - Rs. C-292/04 - Meilicke, EuGHE 2007, I-1835.

108 EuGH, Urt. v. 6.3.2007 - Rs. C-292/04 - Meilicke, EuGHE 2007, I-1835 - Rz. $32 \mathrm{ff}$.

109 Unten IV 1.

110 Unten IV 2.

111 Unten IV 3.

$112 \mathrm{Zu}$ den Konsequenzen im Grundrechtsbereich statt aller Di Fabio in Maunz/ Dürig, GG, Art. 2 Abs. 1 GG Rz. 44. 
Darin liegt der markanteste Unterschied zwischen Entscheidungen des Bundesverfassungsgerichts und solchen EuGH. Aus diesem Unterschied folgt, dass dem EuGH keine Normverwerfungskompetenz, in diesem Sinne nicht einmal einen Normenkontrollkompetenz zukommt.

Dieser Unterschied verblasst allerdings, wenn man die Frage, wie das Gemeinschaftsrecht wirkt, nicht aus der EG-Perspektive, sondern aus der Perspektive des nationalen Verfassungsrechts betrachtet: Denn auch wenn das EG-Recht (ganz ähnlich wie übrigens das klassische Völkerrecht) lediglich Anwendungsvorrang beansprucht, kann sich aus dem innerstaatlichen Verfassungsrecht doch so etwas wie ein Geltungsvorrang ergeben. Wenn schon für den Bereich völkerrechtlicher Verträge nach einer im Vordringen befindlichen Ansicht gilt, dass der treaty override gegen das im Lichte der Verfassungsentscheidung für die internationale Zusammenarbeit $\mathrm{zu}$ betrachtende Rechtsstaatsprinzip verstößt und damit grundsätzlich verfassungswidrig ist ${ }^{113}$, so muss dies angesichts des Integrationsauftrags in Art. 23 GG für Verstöße gegen Gemeinschaftsrecht, jedenfalls gegen primäres Gemeinschaftsrecht erst recht gelten.

Das Gemeinschaftsrecht begründet eine Rechtstreuepflicht der Mitgliedstaaten, die sich auf alle ihre Staatsorgane erstreckt. Sie lässt zwar den Anwendungsvorrang nicht automatisch zum Geltungsvorrang erstarken, verpflichtet aber zu einer rechtsstaatlichen, insbesondere täuschungsfreien Gesetzgebung. Jedenfalls dann, wenn eine mitgliedstaatliche Norm (einschließlich eines förmlichen Parlamentsgesetzes) nicht nur in Randbereichen, sondern in zentralen oder sogar sämtlichen Anwendungsfällen zwingend zu gemeinschaftsrechtswidrigen Ergebnissen führt, unterliegt bereits der mitgliedstaatliche Normgeber gemeinschaftsrechtlichen Bindungen, näherhin: dem Verbot einer entsprechenden Normsetzung.

Folgt man dem, können unter Umständen gemeinschaftsrechtswidrige Normen des deutschen Steuerrechts großflächig, d.h. auch über den konkreten Anlassfall und sogar über den grenzüberschreitenden Fall

113 Statt aller Klaus Vogel, Wortbruch im Verfassungsrecht, JZ 1997, 161; Klaus Vogel, Völkerrechtliche Verträge und innerstaatliche Gesetzgebung, IStR 2005, 29; Rust/Reimer, Treaty Override im deutschen Internationalen Steuerrecht, IStR 2005, 843; Gosch, Über das Treaty Overriding, IStR 2008, 413; Brombach-Krüger, Treaty Override aus europarechtlicher und verfassungsrechtlicher Sicht, Ubg 2008, 324. 
hinaus nichtig sein, wenn der Wortlaut den grenzüberschreitenden Fall ausdrücklich einschließt und keine gemeinschaftsrechtskonforme Auslegung mehr zulässt. Zu betonen ist nochmals, dass damit prozessual keine Normverwerfungskompetenz des EuGH zu begründen ist. Materiell-faktisch geht die Entwicklung aber in die Richtung einer erheblichen Ausweitung der sachlichen Streubreite seiner Entscheidungen.

\section{Zeitliche Wirkung von Entscheidungen}

Besondere Aufmerksamkeit hat zuletzt das Problem der zeitlichen Wirkung gefunden, die den Entscheidungen des EuGH in Steuersachen beizumessen ist. ${ }^{114}$ Ausgangspunkt aller Überlegungen ist die Grundentscheidung darüber, ob die Rolle des Richters als auf einen Rechtserkenntnisakt beschränkt zu denken ist oder ob man dem Spruchkörper - im Sinne richterrechtlicher Konkretisierungskompetenz - eine derivative Regelungsbefugnis, ein Recht zur Nachverdichtung in den vom Normgeber offen gelassenen Fragen zubilligt.

Der EuGH selbst sieht sich als reine Rechtserkenntnisinstanz und geht folgerichtig davon aus, dass seinen Entscheidungen auch in zeitlicher Hinsicht Wirkungen über den Einzelfall hinaus zukommen. In einer mittlerweile dreißigjährigen Rechtsprechungslinie beansprucht er, grundsätzlich nur auszusprechen, was zwischen den Parteien immer schon galt oder verlangt werden konnte: Durch die Auslegung einer Vorschrift des Gemeinschaftsrechts, die der Gerichtshof in Ausübung seiner Befugnisse aus Art. 234 EG vornimmt, erläutere und verdeutliche er nur, in welchem Sinn und mit welcher Tragweite diese Vor-

114 Hierzu Waldhoff, Rückwirkung von EuGH-Entscheidungen (Schriftenreihe "Vorträge und Berichte“ des Zentrums für Europäisches Wirtschaftsrecht an der Universität Bonn, Bd. 155), Bonn 2006; Kokott/Henze, Die Beschränkung der zeitlichen Wirkung von EuGH-Urteilen in Steuersachen, NJW 2006, 177; Thömmes, Zeitliche Wirkung von EuGH-Urteilen, in JbFfSt 2006/2007, S. 50 ff.; Billig, Beschränkung der zeitlichen Wirkung eines Urteils, FR 2007, 785; Michael Lang, Die Beschränkung der zeitlichen Wirkung von EuGH-Urteilen im Lichte des Urteils Meilicke, IStR 2007, 235; Wiedmann, Zeitlos wie ungeklärt: Die Beschränkung der zeitlichen Wirkung von Urteilen des EuGH im Vorabentscheidungsverfahren nach Art. 234 EG, EuZW 2007, 692. 
schrift seit ihrem Inkrafttreten zu verstehen und anzuwenden ist oder gewesen wäre. 115

Dahinter steht - unausgesprochen, aber nicht unproblematisch 116 - der Glaube an die „one right answer" (Dworkin). Er verknüpft die Grundfrage nach dem Wesen des richterlichen Akts eng mit der soeben117 behandelten Frage nach der persönlichen Streubreite von Entscheidungen: Wenn der EuGH in einer Entscheidung einen Rechtserkenntnisakt sieht, kann diese Entscheidung tendenziell über den anhängigen Einzelfall hinaus Wirkung beanspruchen, während das Konzept des Urteils als eigenständiger Rechtssetzungsakt für eine Begrenzung seiner Wirkungen auf den konkret anhängigen Einzelfall gesprochen hätte.

Der Anspruch auf eine erga-omnes- und erga-omnia-tempora-Wirkung der Rechtsprechung ist zahlreichen Einwänden ausgesetzt. Die hohe Streubreite richterlicher Rechtserkenntnisakte läuft der am Institut der Rechtskraft geschärften und an den prozessualen Einwirkungsmöglichkeiten (nur) der Beteiligten orientierten Beschränkung der Entscheidungswirkungen auf das inter-partes-Verhältnis diametral zuwider. Sie wirft zudem aus demokratietheoretischer Sicht, aber auch im Hinblick auf die Garantie der Unabhängigkeit des (späteren) Richters nicht unerhebliche Schwierigkeiten auf.

Immerhin erklärt diese Selbstfestlegung des EuGH dessen Höchstmaß an Bemühen darum, die Kontinuität der Rechtsprechung zu wahren und Änderung der Rechtsprechung möglichst nicht offen zu legen.

Vor allem aber ergeben sich aus dieser Festlegung wichtige Konsequenzen für die hier zu beantwortende Frage nach den zeitlichen Wirkungen von Entscheidungen des Gerichtshofs. Der EuGH betont in ständiger Rechtsprechung, dass aus dem Charakter seiner Entschei-

115 EuGH, Urt. v. 27.3.1980 - Rs. 61/79 - Amministrazione delle finanze dello Stato gegen Denkavit italiana Srl., EuGHE 1980, 1205 - Rz. 16; EuGH, Urt. v. 2.2.1988 - Rs. 24/86 - Vincent Blaizot, EuGHE 1988, 379 - Rz. 27; EuGH, Urt. v. 4.5.1999 - Rs. C-262/96 - Sema Sürül, EuGHE 1999, I-2685 - Rz. 107; EuGH, Urt. v. 3.10.2002 - Rs. C-347/00 - Ángel Barreira Pérez, EuGHE 2002, I-8191 - Rz. 44; EuGHE, Urt. v. 6.3.2007 - Rs. C-292/04 - Meilicke, EuGHE 2007, I-1835 - Rz. 34.

116 Zur Kritik statt aller Auer, Materialisierung, Flexibilisierung, Richterfreiheit, Tübingen 2005, S. 88 ff.; Rosenfeld, Dworkin and the One Law Principle: A Pluralist Critique, Revue Internationale de Philosophie Vol. 59 (2005), 363.

117 Oben IV 1. 
dungen als bloß deklaratorischen Rechtserkenntnisakten folge, dass die Entscheidungen grundsätzlich 118 auf den Zeitpunkt des Inkrafttretens der ausgelegten Vorschrift zurückwirkten ${ }^{119}$. Es liegt auf dieser Linie, dass der EuGH auch von den mitgliedstaatlichen Behörden und Gerichten erwartet, dass diese das Gemeinschaftsrecht in der Auslegung, die der EuGH ihm gegeben hat, auch auf Rechtsverhältnisse anwenden könnten und müssten, die vor Erlass des auf das Ersuchen um Auslegung ergangenen Urteils entstanden sind.

Nach ständiger Rechtsprechung des EuGH verpflichtet der in Art. 10 EG verankerte Grundsatz der Zusammenarbeit eine Verwaltungsbehörde allerdings nur unter erheblich erhöhten Voraussetzungen an das Bemühen der von früheren Entscheidungen beschwerten Betroffenen um die Durchsetzung ihrer gemeinschaftsrechtlichen Positionen zur Aufhebung oder Änderung einer bestandskräftigen Verwaltungsent-

$118 \mathrm{Zu}$ den Ausnahmen (die an bestimmte materielle, aber auch formelle Anforderungen wie einen rechtzeitigen Antrag und eine Feststellung im jeweiligen Urteil selbst geknüpft werden): EuGH, Urt. v. 8.4.1976 - Rs. 43/75 - Gabrielle Defrenne, EuGHE 1976, 455 - Rz. 69 ff.; EUGH, Urt. v. 27.3.1980 - Rs. 61/79 Amministrazione delle finanze dello Stato gegen Denkavit italiana Srl., EuGHE 1980, 1205 - Rz. 17 ff.; EuGH, Urt. v. 2.2.1988 - Rs. 24/86 - Vincent Blaizot, EuGHE 1988, 379 - Rz. 28 ff.; EuGH, Urt. v. 4.5.1999 - Rs. C-262/96 Sema Sürül, EuGHE 1999, I-2685 - Rz. 108 ff. (dort auch zur Rückausnahme einer "Ergreiferprämie" zugunsten derjenigen, die vor der Entscheidung des EuGH gerichtlich Klage erhoben oder einen gleichwertigen Rechtsbehelf eingelegt haben; siehe hierzu noch unten IV 3); EuGH, Urt. v. 3.10.2002 - Rs. C-347/00 - Ángel Barreira Pérez, EuGHE 2002, I-8191 - Rz. 45 f.; EuGH, Urt. v. 6.3.2007 - Rs. C-292/04 - Meilicke, EuGHE 2007, I-1835 - Rz. 35 ff. Aus der Literatur statt aller Waldhoff, Rückwirkung von EuGH-Entscheidungen (Schriftenreihe "Vorträge und Berichte" des Zentrums für Europäisches Wirtschaftsrecht an der Universität Bonn, Bd. 155), Bonn 2006, S. 29 ff.

119 Ständige Rechtsprechung; siehe hierzu EuGH, Urt. v. 27.3.1980 - Rs. 61/79 Amministrazione delle finanze dello Stato gegen Denkavit italiana Srl., EuGHE 1980, 1205 - Rz. 16; EuGH, Urt. v. 2.2.1988 - Rs. 24/86 - Vincent Blaizot, EuGHE 1988, 379 - Rz. 27; EuGH, Urt. v. 4.5.1999 - Rs. C-262/96 Sema Sürül, EuGHE 1999, I-2685 - Rz. 107; EuGH, Urt. v. 3.10 .2002 - Rs. C347/00 - Ángel Barreira Pérez, EuGHE 2002, I-8191 - Rz. 44; EuGHE, Urt. v. 6.3.2007 - Rs. C-292/04 - Meilicke, EuGHE 2007, I-1835 - Rz. 34; EuGH, Urt. v. 19.10.1995 - Rs. C-137/94 - Richardson, EuGHE 1995, I-3407 - Rz. 33; EuGH, Urt. v. 12.2.2008 - Rs. C-2/06 - Willy Kempter KG gegen Hauptzollamt Hamburg-Jonas, EuGHE 2008, I-411 - Rz. 35 am Ende. 
scheidung. Sie ist nur dann zu revidieren, wenn folgende vier Voraussetzungen kumulativ erfüllt sind:

1. Die Behörde muss nach nationalem Recht befugt sein, diese Entscheidung zurückzunehmen.

2. Der Betroffene muss seinerzeit den Rechtsweg erschöpft haben, d.h. die Entscheidung muss infolge des Urteils eines in letzter Instanz entscheidenden nationalen Gerichts bestandskräftig geworden sein.

3. Das damalige Urteil muss - gemessen an einer späteren Entscheidung des Gerichtshofes - auf einer unrichtigen Auslegung des Gemeinschaftsrechts durch das nationale Gericht beruhen. Dabei kommt es nicht darauf an, ob die Parteien in dem Ausgangsverfahren die betreffende gemeinschaftsrechtliche Frage überhaupt aufgeworfen haben. Es genügt vielmehr, dass der gemeinschaftsrechtliche Gesichtspunkt, dessen Auslegung sich in Anbetracht eines späteren Urteils des Gerichtshofs als unrichtig erwiesen hat, von dem in letzter Instanz entscheidenden nationalen Gericht entweder geprüft wurde oder von Amts wegen hätte aufgegriffen werden können. ${ }^{120}$ Das setzt voraus, dass der Gerichtshof seinerzeit unter Verstoß gegen Art. 234 Abs. 3 EG nicht um eine Vorabentscheidung ersucht wurde.

4. Der Betroffene muss sich, unmittelbar nachdem er Kenntnis von der neuen Entscheidung des Gerichtshofes erlangt hat, an die Verwaltungsbehörde gewandt haben. ${ }^{121}$

Parallel, aber etwas zurückhaltender konstruiert der EuGH die Konsequenzen, die sich für die mitgliedstaatlichen Gerichte aus der Rückwirkung seiner Entscheidungen ergeben. Hier stellt der EuGH die Pflicht zur rückwirkenden (retrospektiven) Umsetzung seiner Entscheidungen unter den Vorbehalt, dass auch alle sonstigen Voraussetzungen für die Anrufung der zuständigen mitgliedstaatlichen Gerichte in einem die Anwendung dieser Vorschriften betreffenden Streit vorliegen.

120 EuGH, Urt. v. 12.2.2008 - Rs. C-2/06 - Willy Kempter KG gegen Hauptzollamt Hamburg-Jonas, EuGHE 2008, I-411 - Rz. 44.

121 EuGH, Urt. v. 13.1.2004 - Rs. C -453/00 - Kühne und Heitz, EuGHE 2004, I-837 - Rz. 26-28; hierzu nunmehr auch EuGH, Urt. v. 12.2.2008 - Rs. C-2/06 - Willy Kempter KG gegen Hauptzollamt Hamburg-Jonas, EuGHE 2008, I-411 - Rz. 54 ff. Zum Ganzen auch Potacs, Bestandskraft staatlicher Verwaltungsakte oder Effektivität des Gemeinschaftsrechts, EuR 2004, 595. 
Zugleich ergeben sich aus Entscheidungen des EuGH Konsequenzen für den mitgliedstaatlichen Gesetzgeber. Nach dem oben Gesagten 122 verpflichtet der Grundsatz der Gemeinschaftstreue einen Mitgliedstaat dazu, Normen, deren Anwendung nicht nur in Randbereichen gemeinschaftsrechtswidrig ist, einer Korrektur auf Rechtssetzungsebene zu unterziehen. In welche Richtung der Gesetzgeber die Diskriminierungen hier aber auflöst - durch Erstreckung der begünstigenden Regelung auf alle, durch Erstreckung der belastenden Regelung auf alle oder durch eine qualitativ andere Neukonzeption - ist weder gemeinschafts- noch verfassungsrechtlich präjudiziert. Es obliegt vielmehr dem im Kern freien Rechtssetzungsermessen des mitgliedstaatlichen Gesetzgebers. ${ }^{123}$

Zum Schutz dieses parlamentarischen Gestaltungsraums müssen tendenziell auch belastende Rückwirkungen verfassungs- und gemeinschaftsrechtlich selbst dann für zulässig erachtet werden, wenn der Gesetzgeber die zunächst einseitig belastende (und daher gemeinschaftsrechtswidrige) Regelung rückwirkend zur allgemeinen Regel macht (so dass sie nicht länger diskriminierend wirkt). Derartige Korrekturen kann der mitgliedstaatliche Gesetzgeber selbst dann, wenn sie sich steuerverschärfend auswirken, für rückwirkend anwendbar erklären. Das hat der I. Senat des Bundesfinanzhofs unlängst - überzeugend - für die Novellierung von § 6 AStG entschieden und damit die Korrektur einer zuvor bestehenden Gemeinschaftsrechtswidrigkeit in den Kanon derjenigen Gesichtspunkte aufgenommen, die für die (ausnahmsweise) Zulässigkeit einer echten Rückwirkung (Rückbewirkung von Rechtsfolgen) sprechen. 124

\section{Umsetzung von EuGH-Entscheidungen im System der innerstaatlichen Korrekturvorschriften}

Abschließend ein kurzer Blick auf die verfahrensrechtlichen Möglichkeiten zur innerstaatlichen Umsetzung von EuGH-Entscheidungen durch die deutschen Finanzbehörden. Dieses breite und facettenreiche

122 Hierzu oben IV 1.

123 Hierzu sehr deutlich Gosch, Kurze, aber grundlegende Nachlese zur Nachlese von Meilicke zu den Schulgeld-Urteilen des EuGH, DStR 2007, 1895 (1896).

124 BFH, Beschl. v. 23.9.2008 - I B 92/08, BFH/NV 2008, $2085=$ DStR 2008, 2154 $=$ IStR 2008, 884 . 
Thema weist enge Bezüge zum Rückwirkungsproblem auf125, erschöpft sich aber nicht darin und kann hier nicht in seinen Einzelheiten entfaltet werden. Hingewiesen sei aber auf einige Grundunterscheidungen:

- Nahezu unproblematisch stellt sich - jedenfalls aus verfahrensrechtlicher Sicht ${ }^{126}$ - die Befolgung der Vorabentscheidungen des EuGH (Art. 234 EG) im jeweiligen Ausgangsfall dar. Hier folgt bereits aus der Garantie effektiven Rechtsschutzes (Art. 19 Abs. 4 GG) in Verbindung mit dem effet utile des Gemeinschaftsrechts, dass der Steuerpflichtige, der als Kläger des Ausgangsverfahrens finanzgerichtlichen Rechtsschutz gesucht hat, die Früchte seines Rechtsbehelfs ernten darf. Dieses Gebot würde sich verfassungsrechtlich möglicherweise relativieren, wenn in die Rechtsprechung des EuGH Tendenzen einer "Ankündigungsrechtsprechung”, Appellentscheidungen oder die Modifikation der Rechtsfolgenaussprüche um Fortgeltungsanordnungen eindringen würden. Solange dies aber - wie bisher ${ }^{127}$ - nicht geschieht (und auch kein ausbrechender Rechtsakt vorliegt), sind alle drei Gewalten von Verfassungs wegen daran gehindert, dem EuGH im konkreten Fall des Ausgangsverfahrens die Gefolgschaft zu verweigern.

Schwieriger ist die Frage nach der Breitenwirkung von EuGH-Entscheidungen auf diejenigen (Veranlagungs-) Fälle, die nicht selbst der Anlass für ein Vorabentscheidungsverfahren nach Art. 234 EG waren. In diese Fallgruppen gehören einerseits behördliche und finanzgerichtliche Parallelverfahren zu Verfahren nach Art. 234 EG, andererseits alle behördlichen und finanzgerichtlichen Verfahren, in denen es auf Rechtsfragen ankommt, die Gegenstand eines Vertragsverletzungsverfahrens i.S.d. Art. 226 EG sind. Für sie alle ist im Anschluss an die oben skizzierten gemeinschaftsrechtlichen Vorgaben ${ }^{128} \mathrm{zu}$ differenzieren.

- Noch offene Parallelverfahren, in denen Steuerpflichtige bereits zu einem Zeitpunkt Rechtsschutz gegen eine als gemeinschaftsrechts-

125 Hierzu Delbrück/Hamacher, Meilicke und die Rückwirkung - Zu § 130 AO bei der praktischen Umsetzung des EuGH-Urteils Meilicke, IStR 2007, 627.

$126 \mathrm{Zu}$ den materiellrechtlichen Irrungen und Wirrungen s. die Bemerkungen am Anfang von Abschnitt IV.

127 Oben IV 2.

128 Oben IV 2. 
widrig angesehene mitgliedstaatliche Regelung gesucht haben, zu dem der EuGH seine Entscheidung noch nicht verkündet hatte, müssen im Sinne der Steuerpflichtigen abgeschlossen werden. Dem liegt der Gedanke der „Ergreiferprämie“ zugrunde. ${ }^{129}$ Der EuGH begründet diese Pflicht mit der Erwägung, dass andernfalls „der gerichtliche Schutz der Rechte, die die einzelnen aus dem Gemeinschaftsrecht herleiten, in nicht gerechtfertigter Weise eingeschränkt würde"130. Dabei stellt der EuGH ausdrücklich klar, dass es nicht zwingend darauf ankommt, dass die Steuerpflichtigen in diesen Parallelverfahren eine gerichtliche Klage erhoben haben. Vielmehr genüge auch ein "gleichwertige[r] Rechtsbehelf"131. Richtigerweise sind darunter - soweit Deutschland betroffen ist - der Einspruch sowie Anträge nach $\S \S 172 \mathrm{ff}$. AO, ferner die Verfassungsbeschwerde $\mathrm{zu}$ verstehen. Es muss sich aber um Hauptsacherechtsbehelfe handeln; AdV-Anträge genügen nicht.

- In anderen Fällen, die am Tag der Veröffentlichung der Entscheidung des EuGH noch nicht in der Sache entschieden waren, ist die neue Rechtsprechung von Gemeinschaftsrechts wegen zu berücksichtigen (effet utile). Der Anwendungsvorrang des Gemeinschaftsrechts lässt diese Pflicht auch auf den innerstaatlichen Bereich durchschlagen. Das ergibt sich aus dem in Art. 23 Abs. 1, Art. 59 Abs. 2 GG und dem Rechtsstaatsprinzip verankerten Grundsatz, dass alle drei Gewalten - auch der Gesetzgeber - gehalten sind, das Gemeinschaftsrecht und die Entscheidungen der Gemeinschaftsorgane jedenfalls insoweit uneingeschränkt und effektiv zur Anwendung zu bringen, als sie sich im Rahmen des Grundsatzes der begrenzten Einzelermächtigung halten, sich also nicht als ausbrechende Akte darstellen. Diese Bindung an die Gemeinschaftsrechtsprechung gilt im Grundsatz für alle drei Gewalten, wenngleich man für die Unter- und Mittelbehörden auch hier die oben

129 EuGH, Urt. v. 4.5.1999 - Rs. C-262/96 - Sema Sürül, EuGHE 1999, I-2685 Rz. 112 f. Zu Herkunft und Sinn dieses Begriffs Schwenke, Ist eine „Ergreiferprämie" nach österreichischem Vorbild sinnvoll?, DStR 1999, 404.

130 EuGH, Urt. v. 4.5.1999 - Rs. C-262/96 - Sema Sürül, EuGHE 1999, I-2685 Rz. 112.

131 EuGH, Urt. v. 4.5.1999 - Rs. C-262/96 - Sema Sürül, EuGHE 1999, I-2685 Rz. 112. 
skizzierten Vorbehalte anbringen kann.132 Besonderer Korrekturvorschriften bedarf es nicht.

- Bereits durchgeführte, aber nach Maßgabe der innerstaatlichen Korrekturvorschriften noch nicht änderungsfeste Veranlagungen sind im Lichte der EuGH-Entscheidung zu überprüfen und - bei positivem Ergebnis - zu ändern. Dieses Gebot folgt ebenfalls aus dem Gemeinschaftsrecht und seinem - verfassungsrechtlich anerkannten - Anwendungsvorrang, für anhängige Rechtsbehelfsverfahren zudem wiederum aus Art. 19 Abs. 4 GG. Einfachgesetzlich schlägt es sich in Vorschriften wie § 165 Abs. 1 Satz 2 Nr. 3 und $\S 363$ Abs. 2 Satz 2 AO nieder. Zumindest die letztgenannte Vorschrift ist wegen Art. 19 Abs. 4 GG meines Erachtens auch verfassungsrechtlich geboten. $\mathrm{Ob}$ das Grundgesetz darüber hinaus auch in anderen Fällen subjektive Ansprüche auf Vorläufigkeitsvermerke oder auf ein Ruhenlassen finanzbehördlicher und -gerichtlicher Verfahren gewährt, bedürfte gesonderter Klärung.

- Für bestandskräftige Steuerbescheide bieten die $\S \S 172$ ff. AO dagegen grundsätzlich keine prinzipale Korrekturmöglichkeit. Eine Änderung kommt hier meines Erachtens nur in Betracht, wenn ein gegenläufiger anderweitiger Fehler hinzutritt, der den Tatbestand einer Korrekturvorschrift i.S.d. $\S \S 172$ ff. erfüllt. In diesem Fall kann die Gemeinschaftsrechtswidrigkeit unter Umständen mitberichtigt werden. Maßgeblich wird regelmäßig § 177 Abs. 1 AO sein.

Erschöpft sind die verwaltungsverfahrensrechtlichen Probleme, die die Umsetzung von Entscheidungen des Europäischen Gerichtshofs mit sich bringt, mit diesen wenigen Grundunterscheidungen bei weitem nicht. Die hier skizzierten Fallgruppen lassen aber bereits erkennen, dass der Druck des Gemeinschaftsrechts und seines effet utile auf das Steuerverfahrensrecht zahlreiche Ansatzpunkte in der Abgabenordnung findet.

\section{Zusammenfassung}

Die Interaktionen zwischen Gemeinschaftsrecht und Verfassungsrecht, zwischen der Rechtsprechung des EuGH und derjenigen des Bundes- 
verfassungsgerichts, sind in den drei hier untersuchten Problemkreisen je unterschiedlich gelagert.

Für das Prozessrecht 133 bleibt es - von Desideraten in Randbereichen abgesehen - bei dem prinzipiell gleichgerichteten Nebeneinander von Gemeinschafts- und Verfassungs(prozess)recht. Die Scharniernorm des Art. 101 Abs. 1 Satz 2 GG effektuiert namentlich den Tatbestand des Art. 234 Abs. 3 EG. Die Rechtswirklichkeit zeigt das Bild eines funktionierenden Kooperationsverhältnisses zwischen dem EuGH und den mitgliedstaatlichen Gerichten, namentlich der deutschen Finanzgerichtsbarkeit.

Im Bereich der materiellen Grenzen der Integration 134 ist dagegen ein strukturelles Gegeneinander von Gemeinschafts- und Verfassungsrecht zu konstatieren. Das Prinzip der begrenzten Einzelermächtigung, die fortbestehende Normsetzungshoheit der Mitgliedstaaten in nichtharmonisierten Rechtsgebieten, aber insbesondere auch das Gebot interorganschaftlicher Rücksichtnahme des EuGH auf den Rat und das dort geltende Einstimmigkeitserfordernis (Art. 94 i.V.m. Art. 95 Abs. 2 EG) gebieten dem EuGH ein nicht geringes Maß an Zurückhaltung bei der Anwendung der Grundfreiheiten auf das Recht der direkten Steuern. Hier hat der EuGH in der Vergangenheit kompetenzwidrige Entscheidungen getroffen. Diese Rechtsfehler stellen sich aber nicht als willkürlich dar und verletzten daher noch nicht spezifisches Verfassungsrecht (Art. 23 Abs. 1 GG).

Im Bereich der Entscheidungsfolgen tritt die Bedeutung des Verfassungsrechts insgesamt weniger deutlich zutage als in den beiden ersten Problemkreisen. Hier erscheinen Verfassungsrecht und Verfassungsprozessrecht weniger als normative Vorgabe denn als Vergleichsfolie und Inspirationsquelle: Denn während die persönliche, sachliche und zeitliche Streubreite von Entscheidungen des Bundesverfassungsgerichts - bei aller Flexibilität der Rechtsfolgenaussprüche heute klare Konturen gefunden hat und auf ein aufnahmebereites Verfahrensrecht trifft, sind die Folgen und die verfahrenstechnische Umsetzung von Entscheidungen des EuGH im deutschen Recht noch nicht in gleichem Maße geklärt. Die Einwirkungen des Verfassungsrechts auf das Steuerverfahrensrecht könnten deshalb Vorbild für die weitere

133 Oben II.

134 Oben III. 
Reinter, Rechtsschutz durch den EuGH: Setzt die deutsche Verfassung Schranken?

Klärung der Einwirkung von EuGH-Entscheidungen auf das Steuerverfahrensrecht werden. 